Proceedings of the Edinburgh Mathematical Society (2005) 48, 337-364 (C)

DOI:10.1017/S0013091504000203 Printed in the United Kingdom

\title{
CLASSIFICATION OF LAGRANGIAN SURFACES OF CONSTANT CURVATURE IN THE COMPLEX EUCLIDEAN PLANE
}

\author{
BANG-YEN CHEN \\ Department of Mathematics, Michigan State University, \\ East Lansing, MI 48824-1027, USA (bychen@math.msu.edu)
}

(Received 1 March 2004)

\begin{abstract}
One of the most fundamental problems in the study of Lagrangian submanifolds from a Riemannian geometric point of view is the classification of Lagrangian immersions of real-space forms into complex-space forms. In this article, we solve this problem for the most basic case; namely, we classify Lagrangian surfaces of constant curvature in the complex Euclidean plane $\mathbb{C}^{2}$. Our main result states that there exist 19 families of Lagrangian surfaces of constant curvature in $\mathbb{C}^{2}$. Twelve of the 19 families are obtained via Legendre curves. Conversely, Lagrangian surfaces of constant curvature in $\mathbb{C}^{2}$ can be obtained locally from the 19 families.
\end{abstract}

Keywords: Lagrangian surface; Legendre curve; surface of constant curvature

2000 Mathematics subject classification: Primary 53D12

Secondary 53C40; 53C42

\section{Introduction}

A submanifold $M$ of a Kaehler manifold $\tilde{M}$ is called Lagrangian if the almost complex structure $J$ of $\tilde{M}$ interchanges each tangent space of $M$ with its corresponding normal space. Lagrangian submanifolds appear naturally in the context of classical mechanics and mathematical physics. For instance, the systems of partial differential equations of Hamilton-Jacobi type led to the study of Lagrangian submanifolds and foliations in the cotangent bundle. Moreover, Lagrangian submanifolds are part of a growing list of mathematically rich special geometries that occur naturally in string theory.

For a Lagrangian submanifold with mean curvature vector $H$ and shape operator $A$, the dual 1-form of $J H$ is the Maslov form. A Lagrangian submanifold is called Maslovian if it has no minimal points and if its Maslov vector field $J H$ is an eigenvector of $A_{H}$.

One of the most fundamental problems in the study of Lagrangian submanifolds from a Riemannian geometric point of view is to classify Lagrangian immersions of real-space forms into complex-space forms. Such a submanifold is either totally geodesic or flat if the immersion is minimal $[\mathbf{1 7}, \mathbf{2 1}]$. For the non-minimal case, Lagrangian submanifolds of constant curvature $c$ in complex-space forms of holomorphic sectional curvature $4 c$ were determined in $[\mathbf{1 9}]$ by using the notion of twisted products. Also, Maslovian 
Lagrangian immersions of real-space forms into complex Euclidean spaces were classified in $[\mathbf{1 2}, \mathbf{1 6}]$. Moreover, Lagrangian surfaces in the complex Euclidean plane $\mathbb{C}^{2}$ have been studied recently by many geometers (see, for example, $[\mathbf{1}-\mathbf{6}, \mathbf{9}, \mathbf{1 2}, \mathbf{1 5}, \mathbf{2 2}, \mathbf{2 3}]$ ). In particular, several important families of Lagrangian surfaces of constant curvature in $\mathbb{C}^{2}$ were constructed in $[\mathbf{3}, \mathbf{9}, \mathbf{1 5}]$. (For the recent survey on Lagrangian submanifolds from a Riemannian point of view, see $[\mathbf{1 0}]$.)

In this article we classify Lagrangian surfaces of constant curvature in the complex Euclidean plane without the Maslovian condition. Our main result states that there are 19 families of such Lagrangian surfaces. Twelve of the 19 families are constructed via Legendre curves. Conversely, Lagrangian surfaces of constant curvature in $\mathbb{C}^{2}$ are locally obtained from these 19 families. As an immediate byproduct, many new examples of Lagrangian surfaces of constant curvature in $\mathbb{C}^{2}$ are discovered.

\section{Preliminaries}

Let $M$ be a Lagrangian submanifold in the complex Euclidean $n$-space $\mathbb{C}^{n}$. We denote the Levi-Civita connections of $M$ and $\mathbb{C}^{n}$ by $\nabla$ and $\tilde{\nabla}$, respectively. The formulae of Gauss and Weingarten are given, respectively, by (see [7])

$$
\left.\begin{array}{c}
\tilde{\nabla}_{X} Y=\nabla_{X} Y+h(X, Y), \\
\tilde{\nabla}_{X} \xi=-A_{\xi} X+D_{X} \xi,
\end{array}\right\}
$$

for tangent vector fields $X$ and $Y$ and normal vector field $\xi$, where $D$ is the connection on the normal bundle. The second fundamental form $h$ is related to the shape operator $A_{\xi}$ by $\langle h(X, Y), \xi\rangle=\left\langle A_{\xi} X, Y\right\rangle$. The mean curvature vector $H$ of $M$ is defined by $H=$ $(1 / n) \operatorname{Tr} h$, where $n=\operatorname{dim} M$. A point $p \in M$ is called minimal if $H$ vanishes at $p$.

For vector fields $X, Y, Z$ and $W$ tangent to the Lagrangian submanifold, we have (see $[\mathbf{1 7}])$

$$
\left.\begin{array}{c}
D_{X} J Y=J \nabla_{X} Y, \\
\langle h(X, Y), J Z\rangle=\langle h(Y, Z), J X\rangle=\langle h(Z, X), J Y\rangle .
\end{array}\right\}
$$

The equations of Gauss and Codazzi are given, respectively, by

$$
\begin{aligned}
\langle R(X, Y) Z, W\rangle & =\left\langle A_{h(Y, Z)} X, W\right\rangle-\left\langle A_{h(X, Z)} Y, W\right\rangle, \\
(\nabla h)(X, Y, Z) & =(\nabla h)(Y, X, Z),
\end{aligned}
$$

where $R$ is the curvature tensor and $\nabla h$ is defined by

$$
(\nabla h)(X, Y, Z)=D_{X} h(Y, Z)-h\left(\nabla_{X} Y, Z\right)-h\left(Y, \nabla_{X} Z\right) .
$$

We recall the following theorems for later use (see $[\mathbf{8}, \mathbf{1 8}])$.

Theorem 2.1. Let $M$ be a simply connected Riemannian n-manifold and let $\sigma$ be a $T M$-valued symmetric bilinear form on $M$ satisfying the following conditions:

(i) $\langle\sigma(X, Y), Z\rangle$ is totally symmetric, 
(ii) $(\nabla \sigma)(X, Y, Z)=\nabla_{X} \sigma(Y, Z)-\sigma\left(\nabla_{X} Y, Z\right)-\sigma\left(Y, \nabla_{X} Z\right)$ is totally symmetric; and

(iii) $R(X, Y) Z=\sigma(\sigma(Y, Z), X)-\sigma(\sigma(X, Z), Y)$.

There then exists a Lagrangian isometric immersion $L: M \rightarrow \mathbb{C}^{n}$ whose second fundamental form $h$ is given by $h=J \sigma$.

Theorem 2.2. Let $L_{1}, L_{2}: M \rightarrow \mathbb{C}^{n}$ be Lagrangian isometric immersions of a Riemannian $n$-manifold with second fundamental forms $h^{1}$ and $h^{2}$, respectively. If

$$
\left\langle h^{1}(X, Y), J L_{1 \star} Z\right\rangle=\left\langle h^{2}(X, Y), J L_{2 \star} Z\right\rangle
$$

for all vector fields $X, Y, Z$ tangent to $M$, then there exists a bi-holomorphic isometry $\phi$ of $\mathbb{C}^{n}$ such that $L_{1}=L_{2} \circ \phi$.

\section{Legendre curves}

A curve $z=z(t)$ is called regular if its speed $v(t):=\left|z^{\prime}(t)\right|$ is nowhere zero. A regular curve $z: I \rightarrow S^{3}(r) \subset \mathbb{C}^{2}$ in the hypersphere $S^{3}(r)$ of radius $r$ centred at the origin of $\mathbb{C}^{2}$ is called Legendre if $\left\langle z^{\prime}(t), \mathrm{i} z(t)\right\rangle=0$ identically. It is known that a unit-speed Legendre curve $z(s)$ in $S^{3}(r) \subset \mathbb{C}^{2}$ satisfies $z^{\prime \prime}(s)=\mathrm{i} \kappa(s) z^{\prime}(s)-z(s) / r^{2}$, where $\kappa(s)$ is the curvature function of $z$ in $S^{3}(1)$ (see $[\mathbf{8}]$ ).

A unit-speed curve $z(s)$ in $S^{3}(r)$ satisfies $z^{\prime \prime}(s)=-z(s) / r^{2}$ if and only if it is a geodesic. A geodesic in $S^{3}(r)$ can be either Legendre or non-Legendre. For example, $z(s)=(\cos s, \sin s)$ is Legendre and $z(s)=\left(\mathrm{e}^{\mathrm{i} s}, 0\right)$ is non-Legendre in $S^{3}(1) \subset \mathbb{C}^{2}$.

For a regular curve in $S^{3}(r)$ with speed $v$, we have the following.

Lemma 3.1. For a positive number $r$, the following results hold.

(1) If $z: I \rightarrow S^{3}(r) \subset \mathbb{C}^{2}$ is a Legendre curve, it satisfies

$$
z^{\prime \prime}(t)=\mathrm{i} \lambda(t) z^{\prime}(t)+\frac{v^{\prime}}{v} z^{\prime}(t)-\frac{v^{2}}{r^{2}} z(t)
$$

where $\lambda=\kappa v$ with $\kappa$ being the curvature of $z$ in $S^{3}(1)$.

(2) Conversely, if a regular curve $z=z(t)$ in $S^{3}(r) \subset \mathbb{C}^{2}$ satisfying (3.1) for some nowhere-zero real-valued function $\lambda$, then $z=z(t)$ is a Legendre curve.

Proof. (1) If $z: I \rightarrow S^{3}(r) \subset \mathbb{C}^{2}$ is a Legendre curve, we have $\left\langle z, \mathrm{i} z^{\prime}\right\rangle=0$. Since $|z|=r$ is constant, we also have $\left\langle z, z^{\prime}\right\rangle=0$. Thus, $z, \mathrm{i} z, z^{\prime}$ and $\mathrm{i} z^{\prime}$ are mutually orthogonal. By differentiating $\left\langle z^{\prime}, \mathrm{i} z\right\rangle=0$, we find $\left\langle z^{\prime \prime}, \mathrm{i} z\right\rangle=0$. Hence we have $z^{\prime \prime}=\mathrm{i} \lambda(t) z^{\prime}+\mu z^{\prime}+\varphi z$ for some real-valued functions $\lambda, \mu, \varphi$. From the identity $\left\langle z^{\prime \prime}, z\right\rangle+v^{2}=\left\langle z^{\prime}, z\right\rangle^{\prime}=0$, we find $\varphi=-v^{2} / r^{2}$. Also, by differentiating $\left\langle z^{\prime}, z^{\prime}\right\rangle=v^{2}$, we have $\left\langle z^{\prime \prime}, z^{\prime}\right\rangle=v v^{\prime}$, which implies $\mu=v^{\prime} / v$. Further, after reparametrization of the Legendre curve by its arc length, we find $\lambda=\kappa v$. Consequently, we have (3.1). 
(2) Conversely, let $z(t)$ be a regular curve in $S^{3}(r)$ satisfying (3.1) for some real-valued nowhere-zero function $\lambda$. It follows from $\langle z, z\rangle=r^{2}$ that $\left\langle z^{\prime \prime}, z\right\rangle=-v^{2}$. Also, from $\left\langle z^{\prime}, z^{\prime}\right\rangle=v^{2}$, we have $\left\langle z^{\prime \prime}, z^{\prime}\right\rangle=v v^{\prime}$. Combining these with (3.1) yields $\left\langle\mathrm{i} z^{\prime}, z\right\rangle=0$. Hence, $z$ is a Legendre curve in $S^{3}(r)$.

Let

$$
S^{2}\left(\frac{1}{2}\right)=\left\{\left(x_{1}, x_{2}, x_{3}\right) \in \mathbb{R}^{3}: x_{1}^{2}+x_{2}^{2}+x_{3}^{2}=\frac{1}{4}\right\} .
$$

Then the Hopf fibration $\pi: S^{3}(1) \rightarrow C P^{1}(4) \equiv S^{2}\left(\frac{1}{2}\right)$ is

$$
\pi(z, w)=\left(z \bar{w} ; \frac{1}{2}\left(|z|^{2}-|w|^{2}\right)\right)
$$

for $(z, w) \in S^{3}(1) \subset \mathbb{C}^{2}$.

For each Legendre curve $\gamma=\gamma(t)$ in $S^{3}(1) \subset \mathbb{C}^{2}$, the projection $\pi \circ \gamma$ is a curve in $S^{2}\left(\frac{1}{2}\right)$. Conversely, each curve $\xi$ in $S^{2}\left(\frac{1}{2}\right)$ gives rise to a horizontal lift $\tilde{\xi}$ in $S^{3}(1)$ via $\pi$, which is unique up to a factor $\mathrm{e}^{\mathrm{i} \theta}, \theta \in \mathbb{R}$. Each horizontal lift of $\xi$ is a Legendre curve in $S^{3}(1)$. Since the Hopf fibration $\pi$ is a Riemannian submersion, each Legendre curve $\gamma$ in $S^{3}$ is projected to a curve $\xi$ in $S^{2}\left(\frac{1}{2}\right)$ with the same curvature function.

\section{Some existence results}

We need the following results in the next section.

Proposition 4.1. Let $\mu=\mu(u, v)$ and $\Phi=\Phi(u, v)$ be real-valued functions defined on a simply connected open subset $U$ of $\mathbb{R}^{2}$ which satisfy

$$
\left.\begin{array}{c}
\frac{\partial \Phi}{\partial u}=\frac{\partial \mu^{2}}{\partial v} \neq 0 \\
\left(\mu_{v} \Phi^{-1} \sqrt{K-\mu^{2}}\right)_{v}+\left(\frac{2\left(K-\mu^{2}\right) \mu_{v}+\Phi \mu_{u}}{\left(K-\mu^{2}\right)^{3 / 2}}\right)_{u}=\frac{-\mu \Phi K}{\sqrt{K-\mu^{2}}},
\end{array}\right\}
$$

where $K$ is a real number such that $K \geqslant \mu^{2}$. Then $P_{\mu \Phi}^{K}:=\left(U, g_{0}\right)$ equipped with the metric

$$
g_{0}=\mu^{2} \mathrm{~d} u^{2}+\frac{\Phi^{2}}{K-\mu^{2}} \mathrm{~d} v^{2}
$$

has constant curvature $K$. Moreover, up to rigid motions, there exists a unique Lagrangian isometric immersion $H_{\mu \Phi}^{K}: P_{\mu \Phi}^{K} \rightarrow \mathbb{C}^{2}$, whose second fundamental form satisfies

$$
\left.\begin{array}{l}
h\left(\frac{\partial}{\partial u}, \frac{\partial}{\partial u}\right)=\left(K+\mu^{2}\right) J \frac{\partial}{\partial u}, \\
h\left(\frac{\partial}{\partial u}, \frac{\partial}{\partial v}\right)=\mu^{2} J \frac{\partial}{\partial v}, \\
h\left(\frac{\partial}{\partial v}, \frac{\partial}{\partial v}\right)=\left(\frac{\Phi}{K-\mu^{2}}\right) J \frac{\partial}{\partial u}+2 \Phi J \frac{\partial}{\partial v} .
\end{array}\right\}
$$


Proof. A direct computation shows that the Levi-Civita connection of $P_{\mu \Phi}^{K}$ satisfies

$$
\left.\begin{array}{l}
\nabla_{\partial / \partial u} \frac{\partial}{\partial u}=(\ln \mu)_{u} \frac{\partial}{\partial u}-\frac{\left(K-\mu^{2}\right) \mu \mu_{v}}{\Phi^{2}} \frac{\partial}{\partial v}, \\
\nabla_{\partial / \partial u \frac{\partial}{\partial v}}=(\ln \mu)_{v} \frac{\partial}{\partial u}+\left(\frac{\mu \mu_{u}}{K-\mu^{2}}+(\ln \Phi)_{u}\right) \frac{\partial}{\partial v}, \\
\nabla_{\partial / \partial v \frac{\partial}{\partial v}}=-\frac{\Phi^{2} \mu \mu_{u}+\left(K-\mu^{2}\right) \Phi \Phi_{u}}{\mu^{2}\left(K-\mu^{2}\right)^{2}} \frac{\partial}{\partial u}+\left(\frac{\mu \mu_{v}}{K-\mu^{2}}+(\ln \Phi)_{v}\right) \frac{\partial}{\partial v},
\end{array}\right\}
$$

and the Gauss curvature of $P_{\mu \Phi}^{K}$ is the positive constant $K$. If we define a symmetric bilinear form $\sigma$ on $P_{\mu \Phi}^{K}$ by

$$
\left.\begin{array}{l}
\sigma\left(\frac{\partial}{\partial u}, \frac{\partial}{\partial u}\right)=\left(K+\mu^{2}\right) \frac{\partial}{\partial u}, \\
\sigma\left(\frac{\partial}{\partial u}, \frac{\partial}{\partial v}\right)=\mu^{2} \frac{\partial}{\partial v}, \\
\sigma\left(\frac{\partial}{\partial v}, \frac{\partial}{\partial v}\right)=\left(\frac{\Phi}{K-\mu^{2}}\right) \frac{\partial}{\partial u}+2 \Phi \frac{\partial}{\partial v},
\end{array}\right\}
$$

then it follows from (4.1), (4.3), (4.4) and the definition of $g_{0}$ that $\langle\sigma(X, Y), Z\rangle$ and $(\nabla \sigma)(X, Y, Z)$ are totally symmetric in $X, Y, Z$. A straightforward computation shows that the curvature tensor $R$ and $\sigma$ satisfy condition (iii) of Theorem 2.1. Thus, Theorems 2.1 and 2.2 imply that, up to rigid motions, there exists a unique Lagrangian immersion $H_{\mu \Phi}^{K}: P_{\mu \Phi}^{K} \rightarrow \mathbb{C}^{2}$ whose second fundamental form is given by (4.2).

Proposition 4.2. Let $\mu=\mu(u, v)$ and $\varphi=\varphi(u, v)$ be real-valued functions defined on a simply connected open subset $U$ of $\mathbb{R}^{2}$ which satisfy

$$
\mu_{v}=\frac{K \varphi_{u}+\varphi \mu \mu_{u}-\mu^{2} \varphi_{u}}{\mu\left(4 K-4 \mu^{2}-\varphi^{2}\right)^{3 / 2}} \neq 0, \quad\left(\frac{G_{u}}{\mu}\right)_{u}+\left(\frac{\mu_{v}}{G}\right)_{v}=-K \mu G,
$$

where $G=1 / \sqrt{4 K-4 \mu^{2}-\varphi^{2}}$ and $K$ is a real number greater than $4 \mu^{2}+\varphi^{2}$. Then $M_{\mu \varphi}^{K}:=\left(U, g_{1}\right)$ with metric $g_{1}=\mu^{2} \mathrm{~d} u^{2}+G^{2} \mathrm{~d} v^{2}$ has constant curvature K. Moreover, up to rigid motions, there exists a unique Lagrangian isometric immersion $F_{\mu \varphi}^{K}: M_{\mu \varphi}^{K} \rightarrow \mathbb{C}^{2}$ whose second fundamental form satisfies

$$
\left.\begin{array}{l}
h\left(\frac{\partial}{\partial u}, \frac{\partial}{\partial u}\right)=\left(K+\mu^{2}\right) J \frac{\partial}{\partial u}, \\
h\left(\frac{\partial}{\partial u}, \frac{\partial}{\partial v}\right)=\mu^{2} J \frac{\partial}{\partial v}, \\
h\left(\frac{\partial}{\partial v}, \frac{\partial}{\partial v}\right)=\left(\frac{1}{4 K-4 \mu^{2}-\varphi^{2}}\right) J \frac{\partial}{\partial u}+\left(\frac{1}{\sqrt{4 K-4 \mu^{2}-\varphi^{2}}}\right) J \frac{\partial}{\partial v} .
\end{array}\right\}
$$


Proof. A direct computation shows that the Levi-Civita connection of $M_{\mu \varphi}^{K}$ satisfies

$$
\left.\begin{array}{l}
\nabla_{\partial / \partial u} \frac{\partial}{\partial u}=(\ln \mu)_{u} \frac{\partial}{\partial u}-\left(4 K-4 \mu^{2}-\varphi^{2}\right) \mu \mu_{v} \frac{\partial}{\partial v}, \\
\nabla_{\partial / \partial u} \frac{\partial}{\partial v}=(\ln \mu)_{v} \frac{\partial}{\partial u}+\frac{4 \mu \mu_{u}+\varphi \varphi_{u}}{4 K-4 \mu^{2}-\varphi^{2}} \frac{\partial}{\partial v}, \\
\nabla_{\partial / \partial v} \frac{\partial}{\partial v}=-\frac{4 \mu \mu_{u}+\varphi \varphi_{u}}{\mu^{2}\left(4 K-4 \mu^{2}-\varphi^{2}\right)^{2}} \frac{\partial}{\partial u}+\frac{4 \mu \mu_{v}+\varphi \varphi_{v}}{4 K-4 \mu^{2}-\varphi^{2}} \frac{\partial}{\partial v},
\end{array}\right\}
$$

and the Gauss curvature of $M_{\mu \varphi}^{K}$ is the constant $K$.

Next, let us define a symmetric bilinear form $\sigma$ on $M_{\mu \varphi}^{K}$ by

$$
\left.\begin{array}{l}
\sigma\left(\frac{\partial}{\partial u}, \frac{\partial}{\partial u}\right)=\left(K+\mu^{2}\right) \frac{\partial}{\partial u}, \\
\sigma\left(\frac{\partial}{\partial u}, \frac{\partial}{\partial v}\right)=\mu^{2} \frac{\partial}{\partial v}, \\
\sigma\left(\frac{\partial}{\partial v}, \frac{\partial}{\partial v}\right)=\left(\frac{1}{4 K-4 \mu^{2}-\varphi^{2}}\right) \frac{\partial}{\partial u}+\left(\frac{1}{\sqrt{4 K-4 \mu^{2}-\varphi^{2}}}\right) \frac{\partial}{\partial v} .
\end{array}\right\}
$$

It follows from (4.5), (4.7) and (4.8) that $\langle\sigma(X, Y), Z\rangle$ and $(\nabla \sigma)(X, Y, Z)$ are totally symmetric. A direct computation shows that the curvature tensor $R$ and $\sigma$ satisfy condition (iii) of Theorem 2.1. Thus, up to rigid motions, there is a unique Lagrangian immersion $F_{\mu \varphi}^{K}: M_{\mu \varphi}^{K} \rightarrow \mathbb{C}^{2}$ whose second fundamental form is given by (4.6).

Proposition 4.3. Let $\mu=\mu(u, v)$ and $\varphi=\varphi(u, v)$ be real-valued functions defined on a simply connected open subset $U$ of $\mathbb{R}^{2}$ which satisfy

$$
\mu_{v}=\frac{\mu^{2} \varphi_{u}-K \varphi_{u}-\varphi \mu \mu_{u}}{\mu\left(4 \mu^{2}+\varphi^{2}-4 K\right)^{3 / 2}} \neq 0, \quad\left(\frac{G_{u}}{\mu}\right)_{u}+\left(\frac{\mu_{v}}{G}\right)_{v}=-K \mu G
$$

where $G=1 / \sqrt{4 \mu^{2}+\varphi^{2}-K}$ and $K$ is a real number less than $4 \mu^{2}+\varphi^{2}$. Then $N_{\mu \varphi}^{K}:=\left(U, g_{2}\right)$ with metric $g_{2}=\mu^{2} \mathrm{~d} u^{2}+G^{2} \mathrm{~d} v^{2}$ has constant curvature K. Moreover, up to rigid motions, there exists a unique Lagrangian isometric immersion $L_{\mu \varphi}^{K}: N_{\mu \varphi}^{K} \rightarrow \mathbb{C}^{2}$ whose second fundamental form satisfies

$$
\left.\begin{array}{l}
h\left(\frac{\partial}{\partial u}, \frac{\partial}{\partial u}\right)=\left(K+\mu^{2}\right) J \frac{\partial}{\partial u}, \\
h\left(\frac{\partial}{\partial u}, \frac{\partial}{\partial v}\right)=\mu^{2} J \frac{\partial}{\partial v}, \\
h\left(\frac{\partial}{\partial v}, \frac{\partial}{\partial v}\right)=\left(\frac{1}{4 \mu^{2}+\varphi^{2}-4 K}\right) J \frac{\partial}{\partial u}+\left(\frac{1}{\sqrt{4 \mu^{2}+\varphi^{2}-4 K}}\right) J \frac{\partial}{\partial v} \cdot
\end{array}\right\}
$$

Proof. This can be proved in the same way as Proposition 4.2. 


\section{Main theorem}

Theorem 5.1. Up to rigid motions of $\mathbb{C}^{2}$, locally (in a neighbourhood of each point belonging to an open dense subset), every Lagrangian surface of constant curvature in $\mathbb{C}^{2}$ is given by of one of the following 19 families.

(1) A totally geodesic Lagrangian plane.

(2) A flat Lagrangian cylinder over a curve.

(3) A flat Lagrangian surface $L(s, t)=\left(\gamma_{1}(s), \gamma_{2}(t)\right)$ which is the direct product of two regular curves $\gamma_{1}, \gamma_{2}$ in the complex line $\mathbb{C}$.

(4) A flat Lagrangian cone $L(s, t)=s z(t)$ over a unit-speed Legendre curve $z$ in $S^{3}(1) \subset$ $\mathbb{C}^{2}$.

(5) A flat Lagrangian surface defined by $L(s, t)=\mathrm{e}^{\mathrm{i} c s} z(t)$, where $c$ is a positive number and $z=z(t)$ is a unit-speed Legendre curve in $S^{3}(1 / c) \subset \mathbb{C}^{2}$.

(6) A flat Lagrangian surface defined by $L(s, t)=s^{1+\mathrm{i} b} z(t)$, where $b$ is a positive number and $z=z(t)$ is a Legendre curve of constant speed $1 / b$ in $S^{3}\left(1 / \sqrt{1+b^{2}}\right) \subset \mathbb{C}^{2}$.

(7) A flat Lagrangian surface defined by

$$
L(s, t)=s z(t)+\int_{0}^{t} \theta(t) z^{\prime}(t) \mathrm{d} t
$$

where $z: I \rightarrow S^{3}(1) \subset \mathbb{C}^{2}$ is a unit-speed Legendre curve in $S^{3}(1)$ defined on an open interval $I \ni 0$ and $\theta(t)$ is a non-constant real-valued function on $I$.

(8) A flat Lagrangian surface defined by

$$
L(s, t)=\frac{\sqrt{2} \mathrm{e}^{(\mathrm{i}-2 a) s}}{c \sqrt{1+a^{2}}}\left(\cos \left(\sqrt{1+4 a^{2}} t\right), \sin \left(\sqrt{1+4 a^{2}} t\right),\right.
$$

where $a, c$ are real numbers with $c \neq 0$.

(9) A flat Lagrangian surface defined by

$$
\begin{aligned}
L(u, v)=\int_{0}^{u} \rho(u, v) z & (u-v) \mathrm{e}^{\mathrm{i} u} \mathrm{~d} u \\
& -\mathrm{e}^{\mathrm{i} u} \int_{0}^{v} \frac{\rho_{v} z^{\prime}(u-v)}{f^{2}(u-v)} \mathrm{d} v+\int_{0}^{v} \int_{0}^{u} \mathrm{e}^{\mathrm{i} u}(\rho(u, v) z(u-v))_{v} \mathrm{~d} u \mathrm{~d} v,
\end{aligned}
$$

where $f$ is a nowhere-zero real-valued function defined on an open interval $I \ni 0$, $\rho$ is a solution of the wave equation

$$
\rho_{u v}-\frac{f^{\prime}(u-v)}{f(u-v)} \rho_{v}-f^{2}(u-v) \rho=0,
$$

with $\rho_{v} \neq 0$, and $z=z(t): I \rightarrow S^{3}(1) \subset \mathbb{C}^{2}$ is a Legendre curve with $f(t)$ as its speed and $-1 / f(t)$ as its curvature function. 
(10) A Lagrangian surface of positive curvature $b^{2}$ defined by $L(s, t)=\left(1+\mathrm{e}^{2 \mathrm{i} b s}\right) z(t)$, where $c$ is a positive number and $z(t)$ is a Legendre curve with constant speed $\frac{1}{2}$ in $S^{3}(1 / 2 b) \subset \mathbb{C}^{2}$.

(11) A Lagrangian surface of positive curvature $b^{2}$ defined by

$$
L(s, t)=\mathrm{e}^{2 \mathrm{i} b s} z(t)+\int_{0}^{t} z^{\prime}(t) \mathrm{e}^{-2 \mathrm{i} \theta(t)} \mathrm{d} t,
$$

where $b$ is a positive number, $\theta(t)$ is a non-constant real-valued function defined on an open interval $I \ni 0$ and $z: I \rightarrow S^{3}(1 / 2 b) \subset \mathbb{C}^{2}$ is a Legendre curve with speed $\frac{1}{2}$.

(12) A Lagrangian surface of positive curvature $b^{2}$ defined by

$$
L=\frac{\left(\sqrt{b^{2} \cos ^{2}(b s)-c^{2}}+\mathrm{i} b \sin (b s)\right) \cos (b s)}{\exp \left\{\mathrm{i}^{-1} \tan ^{-1}\left(\sin (b s) / \sqrt{b^{2} \cos ^{2}(b s)-c^{2}}\right)\right\}} z(t),
$$

where $b$ and $c$ are positive numbers with $b>c$ and $z(t)$ is a Legendre curve with speed $1 / \sqrt{b^{2}-c^{2}}$ in $S^{3}\left(1 /\left(b^{2}-c^{2}\right)\right)$.

(13) A Lagrangian surface of positive curvature $b^{2}$ defined by

$$
L=\frac{\left(\sqrt{b^{2} \cos ^{2}(b s)+c^{2}}+\mathrm{i} b \sin (b s)\right) \cos (b s)}{\exp \left\{-\mathrm{i} b^{-1} c \tanh ^{-1}\left(c \sin (b s) / \sqrt{b^{2} \cos ^{2}(b s)+c^{2}}\right)\right\}} z(t),
$$

where $b$ and $c$ are positive numbers and $z(t)$ is a Legendre curve with speed $1 / \sqrt{b^{2}+c^{2}}$ in $S^{3}\left(1 /\left(b^{2}+c^{2}\right)\right)$.

(14) A Lagrangian surface of negative curvature $-b^{2}$ defined by

$$
L(s, t)=z(t)\left(b+\mathrm{i} \sqrt{c^{2} \mathrm{e}^{-2 b s}-b^{2}}\right) \exp \left\{2 b s-\mathrm{i} b^{-1} \sqrt{c^{2} \mathrm{e}^{-2 b s}-b^{2}}\right\},
$$

where $b$ and $c$ are positive and $z(t)$ is a Legendre curve with speed $\mathrm{e}^{\theta(t)} / c$ in $S^{3}\left(1 / c^{2}\right)$.

(15) A Lagrangian surface of negative curvature $-b^{2}$ defined by

$$
L=\frac{\left(\sqrt{c^{2}-b^{2} \cosh ^{2}(b s)}-\mathrm{i} b \sinh (b s)\right) \cosh (b s)}{\exp \left\{-\mathrm{i} b^{-1} c \tan ^{-1}\left(c \sinh (b s) / \sqrt{c^{2}-b^{2} \cosh ^{2}(b s)}\right)\right\}} z(t),
$$

where $z(t)$ is a Legendre curve with speed $1 / \sqrt{c^{2}-b^{2}}$ in $S^{3}\left(1 / \sqrt{c^{2}-b^{2}}\right)$ with $c>$ $b>0$.

(16) A Lagrangian surface of negative curvature $-b^{2}$ defined by

$$
L(s, t)=\frac{\left(\sqrt{c^{2}-b^{2} \sinh ^{2}(b s)}-\mathrm{i} b \cosh (b s)\right) \sinh (b s)}{\left\{\mathrm{i}^{-1} c \tanh ^{-1}\left(c \cosh (b s) / \sqrt{c^{2}-b^{2} \sinh ^{2}(b s)}\right)\right.} z(t),
$$

where $z(t)$ is a Legendre curve with constant speed $\sqrt{b^{2}+c^{2}}$ in $S^{3}\left(1 / \sqrt{b^{2}+c^{2}}\right)$. 
(17) A Lagrangian surface $P_{\mu \Phi}^{K}$ given in Proposition 4.1.

(18) A Lagrangian surface $M_{\mu \varphi}^{K}$ given in Proposition 4.2.

(19) A Lagrangian surface $N_{\mu \varphi}^{K}$ given in Proposition 4.3.

Proof. Let $M$ be a Lagrangian surface of constant curvature $K$ in $\mathbb{C}^{2}$. Denote the tangent bundle of $M$ by $T M$. If $M$ is minimal in $\mathbb{C}^{2}$, then it is totally geodesic (see [17]). So $M$ is an open portion of a Lagrangian plane in $\mathbb{C}^{2}$. This gives (1).

Next, let us assume that $M$ is non-minimal. Then $U=\{p \in M: H(p) \neq 0\}$ is a non-empty open subset. In the rest of the proof, we shall work only on $U$. For each point $p \in U$, we define a function $\gamma_{p}$ by $\gamma_{p}: T_{p}^{1} U \rightarrow \mathbb{R}: v \mapsto \gamma_{p}(v)=\langle h(v, v), J v\rangle$, where $T_{p}^{1} U=\left\{v \in T_{p} U:\langle v, v\rangle=1\right\}$. Since $T_{p}^{1} U$ is a unit circle which is compact, there exists a vector $v \in T_{p}^{1} U$ such that $\gamma_{p}$ attains an absolute minimum at $v$. Since $p$ is a non-totally geodesic point, (2.2) implies that $\gamma_{p} \neq 0$. So, by applying linearity, we have $\gamma_{p}(v)<0$. Because $\gamma_{p}$ attains an absolute minimum at $v$, it follows from (2.2) that $\langle h(v, v), J w\rangle=0$ for all $w$ orthogonal to $v$. Thus, by using (2.2), we know that $v$ is an eigenvector of the shape operator $A_{J v}$. Hence, there exists an orthonormal basis $\left\{e_{1}, e_{2}\right\}$ of $T_{p} M$ with $e_{1}=v$ which satisfies

$$
h\left(e_{1}, e_{1}\right)=\lambda J e_{1}, \quad h\left(e_{1}, e_{2}\right)=\mu J e_{2}, \quad h\left(e_{2}, e_{2}\right)=\mu J e_{1}+\varphi J e_{2}
$$

for some functions $\lambda, \mu, \varphi$ with $(\lambda+\mu)^{2}+\varphi^{2}>0$ on $U$. From (5.1) and the equation of Codazzi we find

$$
\left.\begin{array}{rl}
e_{1} \mu & =\varphi \omega_{1}^{2}\left(e_{1}\right)+(\lambda-2 \mu) \omega_{1}^{2}\left(e_{2}\right), \\
e_{2} \lambda & =(\lambda-2 \mu) \omega_{1}^{2}\left(e_{1}\right), \\
e_{1} \varphi & =3 \mu \omega_{1}^{2}\left(e_{1}\right)+\varphi \omega_{1}^{2}\left(e_{2}\right),
\end{array}\right\}
$$

where $\nabla_{X} e_{1}=\omega_{1}^{2}(X) e_{2}$. Also, from (5.1) and the equation of Gauss we have

$$
\lambda \mu-\mu^{2}=K=\text { const. }
$$

Case A. $\varphi=0$. We divide this into several cases.

Case A.1. $(\lambda-\mu) \mu=0$. In this case, the Lagrangian surface is a flat Lagrangian $H$-umbilical surface. Thus we obtain (2) and (7) by the main theorem of [9].

Case A.2. $\lambda=2 \mu \neq 0$. We have (10) and (11) by applying Theorem 1 of [12].

Case A.3. $\mu \neq 0$ and $\lambda \neq \mu, 2 \mu$. We have $K \neq 0$. Since $\varphi=0,(5.2)$ reduces to

$$
e_{1} \mu=(\lambda-2 \mu) \omega_{1}^{2}\left(e_{2}\right), \quad e_{2} \mu=3 \mu \omega_{1}^{2}\left(e_{1}\right), \quad e_{2} \lambda=(\lambda-2 \mu) \omega_{1}^{2}\left(e_{1}\right) .
$$

Differentiating (5.3) with respect to $e_{2}$ and applying (5.4) yield $\omega_{2}^{1}\left(e_{1}\right)=0$. Hence there exists a local coordinate system $\{s, u\}$ on $M$ such that

$$
g=\mathrm{d} s^{2}+G^{2}(s, u) \mathrm{d} u^{2}
$$


for some function $G$ with $\partial / \partial s=e_{1}, \partial / \partial u=G e_{2}$. It follows from (5.4) and $\omega_{1}^{2}\left(e_{1}\right)=0$ that $e_{2} \lambda=e_{2} \mu=0$. Hence we have $\lambda=\lambda(s)$ and $\mu=\mu(s)$. Using (5.5), we find

$$
\nabla_{\partial / \partial u} \frac{\partial}{\partial s}=(\ln G)_{s} \frac{\partial}{\partial u}, \quad \omega_{1}^{2}\left(e_{2}\right)=\frac{G_{s}}{G} .
$$

Thus, by $(5.4)-(5.6)$, we obtain $(\ln G)_{s}=\mu^{\prime} /(\lambda-2 \mu)=\mu \mu^{\prime} /\left(K-\mu^{2}\right)$, which implies that $G=F(u) / \sqrt{\left|K-\mu^{2}\right|}$ for some function $F$. Thus, (5.5) becomes

$$
g=\mathrm{d} s^{2}+\frac{F^{2}(u)}{\left|K-\mu^{2}(s)\right|} \mathrm{d} u^{2} .
$$

If $t$ denotes an anti-derivative of $F(u)$, then we obtain from (5.7) that

$$
g=\mathrm{d} s^{2}+\frac{\mathrm{d} t^{2}}{\left|K-\mu^{2}(s)\right|}, \quad G^{2}(s)=\frac{1}{\left|K-\mu^{2}(s)\right|} .
$$

Case A.3.i. $K=b^{2}>0$. Since $G$ satisfies $G_{s s}+K G=0$, we have $G=c_{1} \cos (b s)+$ $c_{2} \sin (b s)$ for some constants $c_{1}, c_{2}$, not both zero. Thus we obtain $G=r \cos (b s+c)$ for some constants $r \neq 0$ and $c$. So, after applying a suitable translation in $s$ and a suitable dilation in $t$, we obtain $G=\cos (b s)$. Therefore, we obtain $g=\mathrm{d} s^{2}+\cos ^{2}(b s) \mathrm{d} t^{2}$ and

$$
\left.\begin{array}{l}
\nabla_{\partial / \partial s} \frac{\partial}{\partial s}=0, \\
\nabla_{\partial / \partial s} \frac{\partial}{\partial t}=-b \tan (b s) \frac{\partial}{\partial t}, \\
\nabla_{\partial / \partial t} \frac{\partial}{\partial t}=b \sin (b s) \cos (b s) \frac{\partial}{\partial s} .
\end{array}\right\}
$$

Case A.3.i.a. $K=b^{2}>\mu^{2}$ and $b>0$. In this case, we have $b^{2}=\mu^{2}+\sec ^{2}(b s) \geqslant 1$. Without loss of generality, we may assume that

$$
\lambda=\frac{2 b^{2}-\sec ^{2}(b s)}{\sqrt{b^{2}-\sec ^{2}(b s)}}
$$

and $\mu=\sqrt{b^{2}-\sec ^{2}(b s)}$. Thus, by applying (5.1), (5.9) and Gauss's formula, we find

$$
\left.\begin{array}{rl}
L_{s s} & =\mathrm{i} \frac{2 b^{2}-\sec ^{2}(b s)}{\sqrt{b^{2}-\sec ^{2}(b s)}} L_{s}, \\
L_{s t} & =\left(\mathrm{i} \sqrt{b^{2}-\sec ^{2}(b s)}-b \tan (b s)\right) L_{t}, \\
L_{t t} & =\left(\mathrm{i} \sqrt{b^{2} \cos ^{2}(b s)-1}+b \sin (b s)\right) \cos (b s) L_{s} .
\end{array}\right\}
$$

After solving the first two equations of this system, we know that $L$ is congruent to

$$
L=\frac{\left(\sqrt{b^{2} \cos ^{2}(b s)-1}+\mathrm{i} b \sin (b s)\right) \cos (b s)}{\exp \left\{\mathrm{i} b^{-1} \tan ^{-1}\left(\sin (b s) / \sqrt{b^{2} \cos ^{2} b s-1}\right)\right\}} z(t)
$$

for some $\mathbb{C}^{2}$-valued function $F(t)$. Substituting this into the last equation of (5.10) yields $z^{\prime \prime}(t)+\left(b^{2}-1\right) z(t)=0$. Also, from (5.11), we find $|z(t)|=1 /\left(b^{2}-1\right),\left\langle\mathrm{i} z, z^{\prime}\right\rangle=0$ and $\left|z^{\prime}(t)\right|^{2}=1 /\left(b^{2}-1\right)$. Thus $z(t)$ is a Legendre great circle in $S^{3}\left(1 /\left(b^{2}-1\right)\right)$ with speed $1 / \sqrt{b^{2}-1}$. Hence we obtain (12) with $c=1$ and $z$ as a Legendre great circle. 
Case A.3.i.b. $K=b^{2}<\mu^{2}$ and $b>0$. In this case, we have $\mu^{2}-b^{2}=\sec ^{2}(b s)$. Hence we may assume that $\lambda=\left(2 b^{2}+\sec ^{2}(b s)\right) / \sqrt{b^{2}+\sec ^{2}(b s)}$ and $\mu=\sqrt{b^{2}+\sec ^{2}(b s)}$. Thus we obtain from (5.1), (5.9) and Gauss's formula that

$$
\left.\begin{array}{rl}
L_{s s} & =\mathrm{i} \frac{2 b^{2}+\sec ^{2}(b s)}{\sqrt{b^{2}+\sec ^{2}(b s)}} L_{s}, \\
L_{s t} & =\left(\mathrm{i} \sqrt{b^{2}+\sec ^{2}(b s)}-b \tan (b s)\right) L_{t}, \\
L_{t t} & =\left(\mathrm{i} \sqrt{b^{2} \cos ^{2}(b s)+1}+b \sin (b s)\right) \cos (b s) L_{s} .
\end{array}\right\}
$$

After solving this system in the same way as in Case A.3.i.a, we obtain (13) with $c=1$ and $z$ as a Legendre great circle.

Case A.3.ii. $K=-b^{2}<0$ and $b>0$. Since $G$ satisfies $G_{s s}+K G=0$, we obtain $G=c_{1} \cosh (b s)+c_{2} \sinh (b s)$ for some constants $c_{1}, c_{2}$, not both zero. Thus, by applying a suitable translation in $s$ and a suitable dilation in $t$, we obtain

$$
g=\mathrm{d} s^{2}+\cosh ^{2}(b s) \mathrm{d} t^{2}, \quad g=\mathrm{d} s^{2}+\sinh ^{2}(b s) \mathrm{d} t^{2} \quad \text { or } \quad g=\mathrm{d} s^{2}+\mathrm{e}^{2 b t} \mathrm{~d} t^{2} .
$$

Case A.3.ii.a. $g=\mathrm{d} s^{2}+\cosh ^{2}(b s) \mathrm{d} t^{2}$. From (5.8) we find $\operatorname{sech}^{2} b s=b^{2}+\mu^{2}$. Thus we have $1 \geqslant \operatorname{sech}^{2} b s \geqslant b^{2}$ and $\mu= \pm \sqrt{\operatorname{sech}^{2} b s-b^{2}}$. Without loss of generality, we may assume that

$$
\lambda=\frac{\operatorname{sech}^{2}(b s)-2 b^{2}}{\sqrt{\operatorname{sech}^{2}(b s)-b^{2}}} \quad \text { and } \quad \mu=\sqrt{\operatorname{sech}^{2}(b s)-b^{2}} .
$$

Thus, by applying (5.1) and Gauss's formula, we obtain

$$
\left.\begin{array}{rl}
L_{s s} & =\mathrm{i} \frac{\operatorname{sech}^{2}(b s)-2 b^{2}}{\sqrt{\operatorname{sech}^{2}(b s)-b^{2}}} L_{s}, \\
L_{s t} & =\left(\mathrm{i} \sqrt{\operatorname{sech}^{2}(b s)-b^{2}}+b \tanh (b s)\right) L_{t}, \\
L_{t t} & =\left(\mathrm{i} \sqrt{1-b^{2} \cosh ^{2}(b s)}-b \sinh (b s)\right) \cosh (b s) L_{s} .
\end{array}\right\}
$$

Solving this system gives (15) with $c=1$ and $z$ being a Legendre great circle.

Case A.3.ii.b. $g=\mathrm{d} s^{2}+\sinh ^{2}(b s) \mathrm{d} t^{2}$. From (5.8) we find $\operatorname{cosech}^{2} b s=b^{2}+\mu^{2}$. Thus we obtain

$$
\mu= \pm \sqrt{\operatorname{cosech}^{2} b s-b^{2}}
$$

Without loss of generality, we may assume that

$$
\lambda=\frac{\operatorname{cosech}^{2}(b s)-2 b^{2}}{\sqrt{\operatorname{cosech}^{2}(b s)-b^{2}}} \quad \text { and } \quad \mu=\sqrt{\operatorname{cosech}^{2}(b s)-b^{2}} .
$$

Thus we obtain

$$
\left.\begin{array}{rl}
L_{s s} & =\mathrm{i} \frac{\operatorname{cosech}^{2}(b s)-2 b^{2}}{\sqrt{\operatorname{cosech}^{2}(b s)-b^{2}}} L_{s}, \\
L_{s t} & =\left(\mathrm{i} \sqrt{\operatorname{cosech}^{2}(b s)-b^{2}}+b \operatorname{coth}(b s)\right) L_{t}, \\
L_{t t} & =\left(\mathrm{i} \sqrt{1-b^{2} \sinh ^{2}(b s)}-b \cosh (b s)\right) \sinh (b s) L_{s} .
\end{array}\right\}
$$


Solving this system yields (16) with $c=1$ and $z$ being a Legendre great circle.

Case A.3.ii.c. $g=\mathrm{d} s^{2}+\mathrm{e}^{2 b s} \mathrm{~d} t^{2}$. From (5.8) we find $\mu^{2}=\mathrm{e}^{-2 b s}-b^{2}$. Without loss of generality, we may assume that

$$
\lambda=\frac{\mathrm{e}^{-2 b s}-2 b^{2}}{\sqrt{\mathrm{e}^{-2 b s}-b^{2}}} \quad \text { and } \quad \mu=\sqrt{\mathrm{e}^{-2 b s}-b^{2}} .
$$

Thus, by applying (5.1) and Gauss's formula, we obtain

$$
\left.\begin{array}{rl}
L_{s s} & =\mathrm{i} \frac{\mathrm{e}^{-2 b s}-2 b^{2}}{\sqrt{\mathrm{e}^{-2 b s}-b^{2}}} L_{s} \\
L_{s t} & =\left(\mathrm{i} \sqrt{\mathrm{e}^{-2 b s}-b^{2}}+b\right) L_{t}, \\
L_{t t} & =\left(\mathrm{i} \sqrt{\mathrm{e}^{-2 b s}-b^{2}}-b\right) \mathrm{e}^{2 b s} L_{s}
\end{array}\right\}
$$

Solving this system yields (14) with $c=1$ and $z$ being a Legendre great circle.

Case B. $\varphi \neq 0$ on some open subset $V$ of $U$. We divide this into two cases.

Case B.1. $\nabla_{e_{1}} e_{1}=0$ on an open neighbourhood $W$ of a point $p \in V$ of a point. Since $\nabla_{e_{1}} e_{1}=0$, there exists a local coordinate system $\{s, u\}$ on $V$ such that

$$
g=\mathrm{d} s^{2}+G^{2}(s, u) \mathrm{d} u^{2}
$$

with $\partial / \partial s=e_{1}, \partial / \partial u=G e_{2}$. Since $\nabla_{e_{1}} e_{1}=0,(5.2)$ reduces to

$$
e_{1} \mu=(\lambda-2 \mu) \omega_{1}^{2}\left(e_{2}\right), \quad e_{2} \lambda=0, \quad e_{2} \mu-e_{1} \varphi=\varphi \omega_{1}^{2}\left(e_{2}\right) .
$$

Differentiating (5.3) with respect to $e_{2}$ and applying (5.18) give $(\lambda-2 \mu) e_{2} \mu=0$. Thus we have $\lambda=2 \mu$ or $e_{2} \mu=0$ at each point of $W$. If $\lambda=2 \mu$ on some open subset $W_{1} \subset W$, then $K=\mu^{2}$ on $W_{1}$, which implies that $\mu$ is constant on $W_{1}$. So, we also have $e_{2} \mu=0$ on $W_{1}$. Consequently, $e_{2} \mu=0$ holds identically on $W$. Therefore, (5.18) yields

$$
e_{1} \mu=(\lambda-2 \mu) \omega_{1}^{2}\left(e_{2}\right), \quad e_{2} \lambda=e_{2} \mu=0, \quad e_{1} \varphi=-\varphi \omega_{1}^{2}\left(e_{2}\right) .
$$

It follows from (5.19) that $\lambda=\lambda(s)$ and $\mu=\mu(s)$. We also obtain from (5.17) that

$$
\nabla_{\partial / \partial u} \frac{\partial}{\partial s}=(\ln G)_{s} \frac{\partial}{\partial u}, \quad \omega_{1}^{2}\left(e_{2}\right)=\frac{G_{s}}{G} .
$$

By applying $(5.19),(5.17)$ and $(5.20)$, we get $(\ln G)_{s}=-(\ln \varphi)_{s}$ which implies that

$$
g=\mathrm{d} s^{2}+\frac{F^{2}(u)}{\varphi^{2}} \mathrm{~d} u^{2}, \quad e_{1}=\frac{\partial}{\partial s}, \quad e_{2}=\frac{\varphi}{F(u)} \frac{\partial}{\partial u}
$$

for some positive function $F(u)$. Thus, by applying (5.21) and the Gauss equation, we have $\varphi \varphi_{s s}-2 \varphi_{s}^{2}=K \varphi^{2}$. After solving this differential equation, we obtain

$$
\varphi= \begin{cases}A \sec (b s+B) & \text { if } K=b^{2}>0, \\ \frac{A}{c s+B} & \text { if } K=0, \\ A \mathrm{e}^{-(b s+B)}, A \operatorname{sech}(b s+B) \text { or } A \operatorname{cosech}(b s+B) & \text { if } K=-b^{2}<0,\end{cases}
$$


where $b>0, c$ are real numbers and $A(u) \neq 0, B(u)$ are functions on $V_{1}$. Let $t(u)$ be an anti-derivative of $1 / A(u)$. Then (5.21) and (5.22) imply, for some function $\theta(t)$, that

$$
g= \begin{cases}\mathrm{d} s^{2}+\cos ^{2}(b s+\theta(t)) \mathrm{d} t^{2} & \text { if } K=b^{2}>0 \\
\mathrm{~d} s^{2}+(c s+\theta(t))^{2} \mathrm{~d} t^{2} & \text { if } K=0, \\
\mathrm{~d} s^{2}+\mathrm{e}^{2(b s+\theta(t))} \mathrm{d} t^{2} & \\
\begin{array}{c}
\text { or } \mathrm{d} s^{2}+\cosh ^{2}(b s+\theta(t)) \mathrm{d} t^{2} \\
\text { or } \mathrm{d} s^{2}+\sinh ^{2}(b s+\theta(t)) \mathrm{d} t^{2}
\end{array} & \text { if } K=-b^{2}<0 .\end{cases}
$$

Case B.1.i. $\varphi \neq 0$ and $\lambda=2 \mu$ on an open subset $U_{1} \subset W$. In this case, both $\lambda$ and $\mu$ are constant and $K=\mu^{2} \geqslant 0$ on $U_{1}$ according to (5.3).

Case B.1.i.a. $\varphi \neq 0$ and $\lambda=\mu=0$ on a neighbourhood $U_{1,1}$ of a point in $U_{1}$. In this case, we get $K=0$. Thus we obtain from (5.22) and (5.23) that

$$
\left.\begin{array}{c}
g=\mathrm{d} s^{2}+(c s+\theta(t))^{2} \mathrm{~d} t^{2}, \quad \lambda=\mu=0, \quad \varphi=\frac{H(t)}{c s+\theta(t)}, \\
\nabla_{\partial / \partial s} \frac{\partial}{\partial s}=0, \quad \nabla_{\partial / \partial s} \frac{\partial}{\partial t}=\frac{c}{c s+\theta(t)} \frac{\partial}{\partial t}, \\
\nabla_{\partial / \partial t} \frac{\partial}{\partial t}=-c(c s+\theta(t)) \frac{\partial}{\partial s}+\frac{\theta^{\prime}(t)}{c s+\theta(t)} \frac{\partial}{\partial t},
\end{array}\right\}
$$

where $H(t)$ is nowhere zero on $U_{1}$. Without loss of generality, we may assume that $H(t)$ is defined on an open interval $I \ni 0$.

From (5.1), (5.24) and Gauss's formula we have

$$
\left.\begin{array}{l}
L_{s s}=0, \\
L_{s t}=\frac{c L_{t}}{c s+\theta(t)}, \\
L_{t t}=-c(c s+\theta(t)) L_{s}+\left(\mathrm{i} H(t)+\frac{\theta^{\prime}(t)}{c s+\theta(t)}\right) L_{t} .
\end{array}\right\}
$$

If $c=0$, then after solving (5.25) we have $L=c_{1} s+z(t)$, where $c_{1}$ is a vector and $z(t)$ a vector function satisfying $z^{\prime \prime}(t)=\left(\mathrm{i} H(t)+(\ln \theta)^{\prime}(t)\right) z^{\prime}(t)$. From the last equation we obtain

$$
z(t)=c_{0}+c_{2} \int_{0}^{t} \theta(t) \mathrm{e}^{\mathrm{i} f(t)} \mathrm{d} t
$$

where $f(t)$ is an anti-derivative of $H(t)$. Hence, after applying a suitable translation, we get

$$
L(s, t)=c_{1} s+c_{2} \int_{0}^{t} \theta(t) \mathrm{e}^{\mathrm{i} f(t)} \mathrm{d} t .
$$

Since $\left|L_{s}\right|=1,\left|L_{t}\right|^{2}=\theta^{2}(t)$ and $\left\langle L_{s}, L_{t}\right\rangle=\left\langle L_{s}, \mathrm{i} L_{t}\right\rangle=0$, we obtain from (5.26) that $\left|c_{1}\right|=\left|c_{2}\right|=1$ and $\left\langle c_{1}, c_{2}\right\rangle=\left\langle c_{1}, \mathrm{i} c_{2}\right\rangle=0$. Hence, we obtain (3). 
Next, let us assume that $c \neq 0$. Then, after solving the first equation in (5.25), we have $L=z(t) s+Q(t)$ for some functions $z, Q$. We may assume that $z, Q$ are defined on an open interval $I \ni 0$. Substituting this into the second equation in (5.25) yields $c Q^{\prime}(t)=\theta(t) z^{\prime}(t)$. Thus $L$ is congruent to

$$
L(s, t)=z(t) s+\frac{1}{c} \int_{0}^{t} \theta(t) z^{\prime}(t) \mathrm{d} t .
$$

If $\theta$ is constant, we obtain (4) after applying a suitable translation in $s$.

If $\theta$ is non-constant, then (5.27) gives

$$
\left.\begin{array}{rl}
L_{s} & =z(t), \\
L_{t} & =\left(s+\frac{\theta(t)}{c}\right) z^{\prime}(t), \\
L_{t t} & =\frac{\theta^{\prime}(t)}{c} z^{\prime}(t)+\left(s+\frac{\theta(t)}{c}\right) z^{\prime \prime}(t) .
\end{array}\right\}
$$

From (5.24) and (5.28), we obtain $|z(t)|^{2}=1$ and $\left|z^{\prime}(t)\right|=c$. Since $L$ is Lagrangian, (5.28) also implies that $\left\langle z, \mathrm{i} z^{\prime}\right\rangle=0$. After substituting (5.27) into the last equation in (5.25), we find $z^{\prime \prime}(t)-\mathrm{i} H(t) z^{\prime}(t)+c^{2} z(t)=0$. Thus $z(t)$ is a Legendre curve of speed $c$ in $S^{3}(1) \subset \mathbb{C}^{2}$. Therefore, after reparametrization of the Legendre curve by its arc length, we obtain (7).

Case B.1.i.b. $\varphi \neq 0$ and $\lambda=2 \mu \neq 0$ on a neighbourhood $U_{1,2}$ of a point in $U_{1}$. Without loss of generality, we may assume that $\lambda=2 \mu=2 b>0$. So we have $K=b^{2}>0$.

From (5.22) and (5.23) we obtain

$$
\left.\begin{array}{c}
g=\mathrm{d} s \otimes \mathrm{d} s+\cos ^{2}(b s+\theta(t)) \mathrm{d} t \otimes \mathrm{d} t, \quad \varphi=H(t) \sec (b s+\theta(t)), \\
\nabla_{\partial / \partial s} \frac{\partial}{\partial s}=0, \quad \nabla_{\partial / \partial s} \frac{\partial}{\partial t}=-b \tan (b s+\theta(t)) \frac{\partial}{\partial t}, \\
\nabla_{\partial / \partial t} \frac{\partial}{\partial t}=\frac{1}{2} b \sin (2 b s+2 \theta(t)) \frac{\partial}{\partial s}-\theta^{\prime}(t) \tan (b s+\theta(t)) \frac{\partial}{\partial t},
\end{array}\right\}
$$

where $H$ is nowhere zero on $U_{1,2}$. Hence, (5.1), (5.29) and Gauss's formula give

$$
\left.\begin{array}{l}
L_{s s}=2 \mathrm{i} b L_{s}, \\
L_{s t}=\mathrm{i} b \sec (b s+\theta) \mathrm{e}^{\mathrm{i}(b s+\theta)} L_{t}, \\
L_{t t}=\mathrm{i} b \cos (b s+\theta) \mathrm{e}^{-\mathrm{i}(b s+\theta)} L_{s}+\left(\mathrm{i} H(t)-\theta^{\prime} \tan (b s+\theta)\right) L_{t} .
\end{array}\right\}
$$

If $\theta$ is constant, we may choose $\theta=0$ by applying a suitable translation in $s$. Hence, we obtain from (5.29) and (5.30) that $g=\mathrm{d} s^{2}+\cos ^{2}(b s) \mathrm{d} t^{2}$ and

$$
\left.\begin{array}{rl}
L_{s s} & =2 \mathrm{i} b L_{s}, \\
L_{s t} & =\mathrm{i} b \mathrm{e}^{\mathrm{i} b s} \sec (b s) L_{t}, \\
L_{t t} & =\mathrm{i} b \mathrm{e}^{-\mathrm{i} b s} \cos (b s) L_{s}+\mathrm{i} H(t) L_{t} .
\end{array}\right\}
$$


After solving the first two equations in (5.31), we know that $L$ is congruent to $L=$ $\left(\mathrm{e}^{2 \mathrm{i} b s}+1\right) z(t)$ for some vector function $z(t)$. Substituting this into the last equation in (5.31) gives $z^{\prime \prime}(t)=\mathrm{i} H(t) z^{\prime}(t)-b^{2} z(t)$. Thus $z(t)$ is a Legendre curve in $S^{3}(1 / 2 b)$ with speed $\frac{1}{2}$. Hence, after reparametrization of $z(t)$, we obtain (10).

Next, assume that $\theta$ is non-constant. Then, after solving the first equation in (5.30), we obtain $L=A(t) \mathrm{e}^{2 \mathrm{i} b s}+B(t)$. Differentiating this equation with respect to $t$ yields

$$
L_{t}=A^{\prime}(t) \mathrm{e}^{2 \mathrm{i} b s}+B^{\prime}(t) .
$$

Substituting this into the second equation of (5.30) yields $B^{\prime}=\mathrm{e}^{-2 \mathrm{i} \theta} A^{\prime}$. Hence, we get $L_{t}=A^{\prime}(t)\left(\mathrm{e}^{\mathrm{i} b s}+\mathrm{e}^{-2 \mathrm{i} \theta(t)}\right)$. Thus, after applying a suitable translation, we obtain

$$
L=\mathrm{e}^{2 \mathrm{i} b s} z(t)+\int_{0}^{t} z^{\prime}(t) \mathrm{e}^{-2 \mathrm{i} \theta(t)} \mathrm{d} t
$$

where $z(t)=A(t)+c_{1}$ for some vector $c_{1}$. Hence we have

$$
\left.\begin{array}{rl}
L_{s} & =2 \mathrm{i} b \mathrm{e}^{2 \mathrm{i} b s} z, \\
L_{t} & =\left(\mathrm{e}^{2 \mathrm{i} b s}+\mathrm{e}^{-2 \mathrm{i} \theta}\right) z^{\prime}, \\
L_{t t} & =-2 \mathrm{i} \theta^{\prime} \mathrm{e}^{-2 \mathrm{i} \theta} z^{\prime}+\left(\mathrm{e}^{2 \mathrm{i} b s}+\mathrm{e}^{-2 \mathrm{i} \theta}\right) z^{\prime \prime} .
\end{array}\right\}
$$

Since the immersion is Lagrangian, (5.34) gives $\left\langle z, \mathrm{i} z^{\prime}\right\rangle=0$. Also, by substituting (5.33) into the last equation in (5.30) we have $z^{\prime \prime}(t)-\mathrm{i}\left(H(t)+\theta^{\prime}(t)\right) z^{\prime}(t)+b^{2} z(t)=0$. Thus we find $\left|L_{s}\right|^{2}=4 b^{2}|z(t)|^{2}$ and $\left|L_{t}\right|^{2}=4 \cos ^{2}(b s+\theta)\left|z^{\prime}(t)\right|^{2}$. Comparing these with the metric tensor gives $|z|=1 / 2 b$ and $\left|z^{\prime}\right|=\frac{1}{2}$. Hence we obtain (11).

Case B.1.ii. $\varphi \neq 0$ and $\lambda \neq 2 \mu$ on an open subset $U_{2}$.

We divide this case into several cases.

Case B.1.ii.a. $\lambda \neq 0$ and $\mu=0$ on a neighbourhood $U_{2,1}$ of a point in $U_{2}$. From (5.3) and (5.18) we have $K=\omega_{1}^{2}=e_{2} \lambda=e_{1} \varphi=0$. Thus there exists a coordinate system $\{s, t\}$ with $e_{1}=\partial / \partial s$ and $e_{2}=\partial / \partial t$. Hence we get $g=\mathrm{d} s^{2}+\mathrm{d} t^{2}, \lambda=\lambda(s)$ and $\varphi=\varphi(t)$. Therefore, by (5.1) and Gauss's formula, we obtain

$$
L_{s s}=\mathrm{i} \lambda(s) L_{s}, \quad L_{s t}=0, \quad L_{t t}=\mathrm{i} \varphi(t) L_{t} .
$$

After solving this system, we know that $L$ is congruent to

$$
L(s, t)=c_{1} \int^{s} \exp \left(\mathrm{i} \int^{x} \lambda(u) \mathrm{d} u\right) \mathrm{d} x+c_{2} \int^{t} \exp \left(\mathrm{i} \int^{y} \varphi(u) \mathrm{d} u\right) \mathrm{d} y .
$$

Thus, we obtain (3) after choosing suitable initial conditions.

Case B.1.ii.b. $\lambda=\mu \neq 0$ on a neighbourhood $U_{2,2}$ of a point in $U_{2}$. From (5.3) and (5.18) we get $K=0$ and

$$
e_{1}(\ln \mu)=-\omega_{1}^{2}\left(e_{2}\right), \quad e_{2} \lambda=e_{2} \mu=0, \quad e_{1} \varphi=-\varphi \omega_{1}^{2}\left(e_{2}\right) .
$$


Thus, $\lambda$ and $\mu$ depend only on $s$ due to (5.21). Combining the first equation in (5.36) with (5.20) gives $G=F(u) / \mu(s)$. If $t(u)$ is an anti-derivative of $F(u)$, we get $g=$ $\mathrm{d} s^{2}+\mu^{-2}(s) \mathrm{d} t^{2}$, which implies $(1 / \mu)^{\prime \prime}=0$. Thus we obtain, for some constants $a, b$, that

$$
g=\mathrm{d} s^{2}+(a s+b)^{2} \mathrm{~d} t^{2} .
$$

If $a=0$ and $b \neq 0$, we get $\omega_{1}^{2}=0$. Combining this with (5.36) implies that $\varphi=\varphi(t)$ and $\lambda=\mu=c$ for some real number $c \neq 0$. Without loss of generality, we may assume $c>0$. Hence, we obtain from (5.1) and Gauss's formula that

$$
L_{s s}=\mathrm{i} c L_{s}, \quad L_{s t}=\mathrm{i} c L_{t}, \quad L_{t t}=\mathrm{i} c L_{s}+\mathrm{i} b^{-1} \varphi(t) L_{t} .
$$

Solving the first two equations of (5.38) gives $L=\mathrm{e}^{\mathrm{i} c s} z(t)$. So, using $\left\langle L_{s}, L_{t}\right\rangle=0$, we have $\left\langle\mathrm{i} z, z^{\prime}\right\rangle=0,\langle z, z\rangle=1 / c^{2}$. Thus $z(t)$ is a Legendre curve in $S^{3}(1 / c)$. By substituting $L=\mathrm{e}^{\mathrm{i} c s} z(t)$ into the last equation of (5.38), we find $b z^{\prime \prime}(t)-\varphi(t) \mathrm{i} z^{\prime}(t)+b c^{2} z(t)=0$. Hence $z=z(t)$ is of unit speed. Consequently, we obtain (5).

Next, assume that $a \neq 0$. Then, after a suitable translation in $s,(5.37)$ becomes

$$
g=\mathrm{d} s^{2}+a^{2} s^{2} \mathrm{~d} t^{2}, \quad \lambda=\mu=(a s)^{-1} .
$$

Without loss of generality, we may assume $a>0$. From (5.39) we get

$$
\nabla_{\partial / \partial s} \frac{\partial}{\partial s}=0, \quad \nabla_{\partial / \partial s} \frac{\partial}{\partial t}=\frac{1}{s} \frac{\partial}{\partial t}, \quad \nabla_{\partial / \partial t} \frac{\partial}{\partial t}=-a^{2} s \frac{\partial}{\partial s} .
$$

Using the last two equations in (5.36), we get $\varphi=s^{-1} f(t)$ for some function $f(t)$. Hence (5.1), (5.39), (5.40) and Gauss's formula imply that

$$
\left.\begin{array}{rl}
L_{s s} & =\frac{\mathrm{i}}{a s} L_{s}, \\
L_{s t} & =\frac{a+\mathrm{i}}{a s} L_{t}, \\
L_{t t} & =(\mathrm{i}-a) a s L_{s}+\mathrm{i} a f(t) L_{t} .
\end{array}\right\}
$$

Solving the first two equations in (5.41) shows that $L$ is congruent to

$$
L(s, t)=s^{1+\mathrm{i} b} z(t), b=a^{-1} .
$$

It follows from (5.42) that $\left\langle\mathrm{i} z, z^{\prime}\right\rangle=0,|z|^{2}=1 /\left(1+b^{2}\right)$ and $\left|z^{\prime}\right|^{2}=1 / b^{2}$. Hence, $z(t)$ is a Legendre curve in $S^{3}\left(1 / \sqrt{1+b^{2}}\right)$ with speed $1 / b$. Hence we obtain (6).

Case B.1.ii.c. $\lambda \neq \mu$ and $\mu \neq 0$ on an open subset $W_{1} \subset U_{2}$. In this case (5.3) implies that $K \neq 0$. Moreover, from (5.3), (5.19) and (5.20), we find that

$$
\left.\begin{array}{rl}
\lambda & =\mu+\mu^{-1} K, \\
\omega_{2}^{1}\left(e_{2}\right) & =e_{1}\left(\ln \sqrt{\left|K-\mu^{2}\right|}\right)=e_{1}(\ln \varphi)=-e_{1}(\ln G)
\end{array}\right\}
$$

on $W_{1}$, where $G$ is defined by (5.18). Hence, we get

$$
G \sqrt{\left|K-\mu^{2}\right|}=p(t), \quad \varphi G=f(t)
$$

for some real-valued functions $f$ and $p>0$. 
Case B.1.ii.c.1. $K=b^{2}>\mu^{2}>0$ on a neighbourhood $W_{1,1}$ of a point $p \in W_{1}$. We may assume $b>0$. From (5.19), (5.23) and (5.44) we get $g=\mathrm{d} s^{2}+\cos ^{2}(b s+\theta(t)) \mathrm{d} t^{2}$ and

$$
\mu^{2}(s)=b^{2}-p^{2}(t) \sec ^{2}(b s+\theta(t)), \quad \varphi=f(t) \sec (b s+\theta(t))
$$

for some functions $p>0$ and $f$ with $b^{2}>p^{2}$. Differentiating the first equation in (5.45) with respect to $t$ gives

$$
(\ln p(t))^{\prime}=\frac{\partial(\ln \cos (b s+\theta(t)))}{\partial t} .
$$

Hence $p(t)=k(s) \cos (b s+\theta(t))$ for some function $k(s)$. So, by differentiating the last equation with respect to $s$ we find $(\ln k(s))^{\prime}=b \tan (b s+\theta(t))$. Hence, $\theta$ and $p$ are constant. So, we may choose $\theta=0$ by applying a suitable translation in $s$. Therefore, we have $g=\mathrm{d} s^{2}+\cos ^{2}(b s) \mathrm{d} t^{2}$ and

$$
\left.\begin{array}{c}
\lambda=\frac{2 b^{2}-c^{2} \sec ^{2}(b s)}{\sqrt{b^{2}-c^{2} \sec ^{2}(b s)},} \quad \mu=\sqrt{b^{2}-c^{2} \sec ^{2}(b s)}, \quad \varphi=f(t) \sec (b s), \\
\nabla_{\partial / \partial s \frac{\partial}{\partial s}}=0, \quad \nabla_{\partial / \partial s \frac{\partial}{\partial t}=-b \tan (b s) \frac{\partial}{\partial t}} \\
\nabla_{\partial / \partial t} \frac{\partial}{\partial t}=\frac{1}{2} b \sin (2 b s) \frac{\partial}{\partial s},
\end{array}\right\}
$$

where $c=p$ is a positive number. From (5.1), (5.46) and Gauss's formula we obtain

$$
\left.\begin{array}{rl}
L_{s s} & =\mathrm{i} \frac{2 b^{2}-c^{2} \sec ^{2}(b s)}{\sqrt{b^{2}-c^{2} \sec ^{2}(b s)}} L_{s}, \\
L_{s t} & =\left(\mathrm{i} \sqrt{b^{2}-c^{2} \sec ^{2}(b s)}-b \tan (b s)\right) L_{t}, \\
L_{t t} & =\left(b \sin (b s)+\mathrm{i} \sqrt{b^{2} \cos ^{2}(b s)-c^{2}}\right) \cos (b s) L_{s}+\mathrm{i} f L_{t} .
\end{array}\right\}
$$

Solving the first two equations implies that $L$ is congruent to

$$
L=\frac{\left(\sqrt{b^{2} \cos ^{2}(b s)-c^{2}}+\mathrm{i} b \sin (b s)\right) \cos (b s)}{\exp \left\{\mathrm{i} b^{-1} \tan ^{-1}\left(\sin (b s) / \sqrt{b^{2} \cos ^{2}(b s)-c^{2}}\right)\right\}} z(t)
$$

for some vector function $z(t)$. Substituting this into the last equation in (5.47) yields $z^{\prime \prime}(t)-\mathrm{i} f(f) z^{\prime}(t)+\left(b^{2}-c^{2}\right) z(t)=0$. On the other hand, we find from (5.48) that $|z(t)|=1 /\left(b^{2}-c^{2}\right),\left|z^{\prime}(t)\right|^{2}=1 /\left(b^{2}-c^{2}\right)$ and $\left\langle\mathrm{i} z, z^{\prime}\right\rangle=0$. Hence $z(t)$ is a unit-speed Legendre curve in $S^{3}\left(1 /\left(b^{2}-c^{2}\right)\right)$.

Therefore, we obtain (12).

Case B.1.ii.c.2. $K=b^{2}<\mu^{2}$ on a neighbourhood $W_{1,2}$ of a point $p \in W_{1}$. We may assume $b>0$. From (5.23) and (5.44) we get $g=\mathrm{d} s^{2}+\cos ^{2}(b s+\theta(t)) \mathrm{d} t^{2}$ and

$$
\mu^{2}=b^{2}+p^{2}(t) \sec ^{2}(b s+\theta(t)), \quad \varphi=f(t) \sec (b s+\theta(t))
$$

for some functions $p(t)$ and $f(t)$ with $b^{2}>p^{2}>0$. Since $\mu=\mu(s)$, the first equation in (5.49) implies that $p(t) \sec (b s+\theta(t))$ depends only on $s$. So, we know as in Case B.1.ii.c.1 
that both $p(t)$ and $\theta(t)$ are constant. Thus we may choose $\theta=0$ by applying a suitable translation in $s$. Hence, we have $g=\mathrm{d} s^{2}+\cos ^{2}(b s) \mathrm{d} t^{2}$ and

$$
\lambda=\frac{2 b^{2}+c^{2} \sec ^{2}(b s)}{\sqrt{b^{2}+c^{2} \sec ^{2}(b s)}}, \quad \mu=\sqrt{b^{2}+c^{2} \sec ^{2}(b s)}, \quad \varphi=f(t) \sec (b s),
$$

where $c=p$ is positive. By applying (5.1), (5.50) and Gauss's formula, we find

$$
\left.\begin{array}{rl}
L_{s s} & =\mathrm{i} \frac{2 b^{2}+c^{2} \sec ^{2}(b s)}{\sqrt{b^{2}+c^{2} \sec ^{2}(b s)}} L_{s}, \\
L_{s t} & =\left(\mathrm{i} \sqrt{b^{2}+c^{2} \sec ^{2}(b s)}-b \tan (b s)\right) L_{t}, \\
L_{t t} & =\left(b \sin (b s)+\mathrm{i} \sqrt{b^{2} \cos ^{2}(b s)+c^{2}}\right) \cos (b s) L_{s}+\mathrm{i} f L_{t} .
\end{array}\right\}
$$

After solving this system as Case B.1.ii.c.1, we obtain (13).

Case B.1.ii.c.3. $K=-b^{2}>0$ on a neighbourhood $W_{1,3}$ of a point $p \in W_{1}$. We may assume $b>0$. In view of (5.23), we divide this into three cases.

Case B.1.ii.c.3. $\boldsymbol{\alpha} . g=\mathrm{d} s^{2}+\mathrm{e}^{2 b s+2 \theta(t)} \mathrm{d} t^{2}$. From (5.23) and (5.44) we get

$$
\mu^{2}=p^{2}(t) \mathrm{e}^{-2 b s-2 \theta(t)}-b^{2}, \quad \varphi=f(t) \mathrm{e}^{-b s-\theta(t)}
$$

for some functions $p(t)$ and $f(t)$. Since $\mu=\mu(s)$, the first equation in (5.51) implies that $p(t)=c \mathrm{e}^{\theta(t)}$ for some real number $c$. Thus we get $\mu^{2}=c^{2} \mathrm{e}^{-2 b s}-b^{2}$. Without loss of generality, we may assume that $\mu=\sqrt{c^{2} \mathrm{e}^{-2 b s}-b^{2}}$. Hence, by (5.43), we have

$$
\lambda=\frac{c^{2} \mathrm{e}^{-2 b s}-2 b^{2}}{\sqrt{c^{2} \mathrm{e}^{-2 b s}-b^{2}}}, \quad \mu=\sqrt{c^{2} \mathrm{e}^{-2 b s}-b^{2}}, \quad \varphi=f(t) \mathrm{e}^{-b s-\theta(t)} .
$$

Therefore, (5.1), (5.52) and Gauss's formula imply that

$$
\left.\begin{array}{rl}
L_{s s} & =\mathrm{i} \frac{c^{2} \mathrm{e}^{-2 b s}-2 b^{2}}{\sqrt{c^{2} \mathrm{e}^{-2 b s}-b^{2}}} L_{s}, \\
L_{s t} & =\left(\mathrm{i} \sqrt{c^{2} \mathrm{e}^{-2 b s}-b^{2}}+b\right) L_{t}, \\
L_{t t} & =\left(\mathrm{i} \sqrt{c^{2} \mathrm{e}^{-2 b s}-b^{2}}-b\right) \mathrm{e}^{2 b s+2 \theta(t)} L_{s}+\mathrm{i} f(t) L_{t} .
\end{array}\right\}
$$

Solving the first two equations in (5.53) shows that $L$ is congruent to

$$
L(s, t)=z(t)\left(b+\mathrm{i} \sqrt{c^{2} \mathrm{e}^{-2 b s}-b^{2}}\right) \exp \left\{2 b s-\mathrm{i} b^{-1} \sqrt{c^{2} \mathrm{e}^{-2 b s}-b^{2}}\right\}
$$

for some vector function $z(t)$. Substituting this into the last equation of the differential system gives $z^{\prime \prime}(t)-\mathrm{i} f(t) z^{\prime}(t)+c^{2} \mathrm{e}^{2 \theta(t)} z(t)=0$.

On the other hand, by using (5.54), we find $|z(t)|=1 / c^{2},\left|z^{\prime}(t)\right|^{2}=\mathrm{e}^{\theta(t)} / c$ and $\left\langle\mathrm{i} z(t), z^{\prime}(t)\right\rangle=0$. Hence $z(t)$ is a Legendre curve in $S^{3}\left(1 / c^{2}\right)$ with speed $\mathrm{e}^{\theta(t)} / c$. Therefore, we obtain (14). 
Case B.1.ii.c.3. $\beta . g=\mathrm{d} s^{2}+\cosh ^{2}(b s+\theta(t)) \mathrm{d} t^{2}$. From (5.23) and (5.44) we get

$$
\mu^{2}(s)=p^{2}(t) \operatorname{sech}^{2}(b s+\theta(t))-b^{2}, \quad \varphi=f(t) \operatorname{sech}(b s+\theta(t))
$$

for functions $p, f$. The first equation in (5.55) is impossible unless $p$ and $\theta$ are both constant. So we have $\theta=0$ after applying a suitable translation in $s$. Hence, we obtain

$$
\lambda=\frac{c^{2} \operatorname{sech}^{2}(b s)-2 b^{2}}{\sqrt{c^{2} \operatorname{sech}^{2}(b s)-b^{2}}}, \quad \mu=\sqrt{c^{2} \operatorname{sech}^{2}(b s)-b^{2}}, \quad \varphi=f(t) \operatorname{sech}(b s),
$$

where $p=c$. From (5.1), (5.56) and Gauss's formula, we find

$$
\left.\begin{array}{rl}
L_{s s} & =\mathrm{i} \frac{c^{2} \operatorname{sech}^{2}(b s)-2 b^{2}}{\sqrt{c^{2} \operatorname{sech}^{2}(b s)-b^{2}}} L_{s}, \\
L_{s t} & =\left(\mathrm{i} \sqrt{c^{2} \operatorname{sech}^{2}(b s)-b^{2}}+b \tanh (b s)\right) L_{t}, \\
L_{t t} & =\left(\mathrm{i} \sqrt{c^{2}-b^{2} \cosh ^{2}(b s)}-b \sinh (b s)\right) \cosh (b s) L_{s}+\mathrm{i} f L_{t} .
\end{array}\right\}
$$

Solving the first two equations of (5.57) shows that $L$ is congruent to

$$
L(s, t)=\frac{\left(\sqrt{c^{2}-b^{2} \cosh ^{2}(b s)}-\mathrm{i} b \sinh (b s)\right) \cosh (b s)}{\exp \left\{-\mathrm{i} b^{-1} c \tan ^{-1}\left(c \sinh (b s) / \sqrt{c^{2}-b^{2} \cosh ^{2}(b s)}\right)\right\}} z(t)
$$

for some vector function $z(t)$. Substituting this into the last equation of (5.57) gives $z^{\prime \prime}(t)-\mathrm{i} f(t) z^{\prime}(t)+\left(c^{2}-b^{2}\right) z(t)=0$.

On the other hand, by using (5.58), we find $|z(t)|=1 /\left(c^{2}-b^{2}\right),\left|z^{\prime}(t)\right|^{2}=1$ and $\left\langle\mathrm{i} z(t), z^{\prime}(t)\right\rangle=0$. Hence $z(t)$ is a unit-speed Legendre curve in $S^{3}\left(1 / \sqrt{c^{2}-b^{2}}\right)$. Consequently, we obtain (15).

Case B.1.ii.c.3. $\gamma \cdot g=\mathrm{d} s^{2}+\sinh ^{2}(b s+\theta(t)) \mathrm{d} t^{2}$. From (5.23) and (5.44) we get

$$
\mu^{2}=p^{2}(t) \operatorname{cosech}^{2}(b s+\theta(t))-b^{2}, \quad \varphi=f(t) \operatorname{cosech}(b s+\theta(t))
$$

for functions $p, f$. As in Case B.1.ii.c.3. $\alpha$, both $p$ and $\theta$ are constant. So, we have $\theta=0$ after applying a suitable translation in $s$. So, if we denote $p$ by $c$, we obtain

$$
\lambda=\frac{c^{2} \operatorname{cosech}^{2}(b s)-2 b^{2}}{\sqrt{c^{2} \operatorname{cosech}^{2}(b s)-b^{2}}}, \quad \mu=\sqrt{c^{2} \operatorname{cosech}^{2}(b s)-b^{2}}, \quad \varphi=f(t) \operatorname{cosech}(b s) .
$$

From (5.1), (5.60) and Gauss's formula, we find

$$
\left.\begin{array}{rl}
L_{s s} & =\mathrm{i} \frac{c^{2} \operatorname{cosech}^{2}(b s)-2 b^{2}}{\sqrt{c^{2} \operatorname{cosech}^{2}(b s)-b^{2}}} L_{s}, \\
L_{s t} & =\left(\mathrm{i} \sqrt{c^{2} \operatorname{cosech}^{2}(b s)-b^{2}}+b \operatorname{coth}(b s)\right) L_{t}, \\
L_{t t} & =\left(\mathrm{i} \sqrt{c^{2}-b^{2} \sinh ^{2}(b s)}-b \cosh (b s)\right) \sinh (b s) L_{s}+\mathrm{i} f L_{t} .
\end{array}\right\}
$$


Solving the first two equations in (5.53) shows that $L$ is congruent to

$$
L(s, t)=\frac{\left(\sqrt{c^{2}-b^{2} \sinh ^{2}(b s)}-\mathrm{i} b \cosh (b s)\right) \sinh (b s)}{\exp \left\{\mathrm{i} b^{-1} c \tanh ^{-1}\left(c \cosh (b s) / \sqrt{c^{2}-b^{2} \sinh ^{2}(b s)}\right)\right\}} z(t)
$$

for a vector function $z(t)$. By substituting this into the last equation of (5.61), we obtain $z^{\prime \prime}(t)-\mathrm{i} f(t) z^{\prime}(t)+\left(b^{2}+c^{2}\right) z(t)=0$.

On the other hand, by applying (5.62), we find $|z(t)|=1 /\left(b^{2}+c^{2}\right)^{2},\left|z^{\prime}(t)\right|^{2}=b^{2}+c^{2}$ and $\left\langle\mathrm{i} z(t), z^{\prime}(t)\right\rangle=0$. Hence, $z(t)$ is a Legendre curve in $S^{3}\left(1 / \sqrt{b^{2}+c^{2}}\right)$ with constant speed $\sqrt{b^{2}+c^{2}}$. Therefore, we obtain (16).

Case B.2. $\nabla_{e_{1}} e_{1} \neq 0$ on an open subset $V_{2} \subset U$. We have $\omega_{1}^{2}\left(e_{1}\right) \neq 0$ on $V_{2}$. Since $\operatorname{span}\left\{e_{1}\right\}$ and $\operatorname{span}\left\{e_{2}\right\}$ are of rank one, there exist coordinates $\{x, y\}$ such that $\partial / \partial x$, $\partial / \partial y$ are parallel to $e_{1}$ and $e_{2}$, respectively. Thus, the metric tensor $g$ takes the form

$$
g=E^{2} \mathrm{~d} x^{2}+G^{2} \mathrm{~d} y^{2}
$$

for some positive functions $E, G$. If we put $\partial / \partial x=E e_{1}, \partial / \partial y=G e_{2},(5.63)$ yields

$$
\omega_{2}^{1}\left(e_{1}\right)=\frac{E_{y}}{E G}, \quad \omega_{1}^{2}\left(e_{2}\right)=\frac{G_{x}}{E G}, \quad E_{y}=\frac{\partial E}{\partial y}, \quad G_{x}=\frac{\partial G}{\partial x} .
$$

If $\lambda=2 \mu$ on some neighbourhood $V_{2,1}$ of a point $p \in V_{2}$, then (5.3) gives $K=\mu^{2}$, which implies that $\mu$ is constant. Thus (5.2) and $\omega_{1}^{2}\left(e_{1}\right) \neq 0$ give $\varphi=0$, which contradicts the assumption $\varphi \neq 0$ for Case B. Hence we must have $\lambda \neq 2 \mu$. Therefore, we obtain $e_{2} \lambda \neq 0$ on $V_{2}$ due to $\omega_{1}^{2}\left(e_{1}\right) \neq 0$ and the second equation in (5.2). So, $\lambda$ is not constant.

Case B.2.i. $\mu=0$ on an open subset $W_{1} \subset V_{2}$. We have $K=0$ from (5.3). Also, we may assume $\lambda, \varphi \neq 0$, otherwise it reduces to Case A.1. From $\mu=0$ and (5.2), we find

$$
\varphi \omega_{1}^{2}\left(e_{1}\right)=\lambda \omega_{2}^{1}\left(e_{2}\right), \quad e_{2} \lambda=\lambda \omega_{1}^{2}\left(e_{1}\right), \quad e_{1} \varphi=\varphi \omega_{2}^{1}\left(e_{2}\right) .
$$

It follows from (5.64) and (5.65) that $\lambda E=\eta(x)$ and $\varphi G=k(y)$ for some functions $\eta(x)$ and $k(y)$. Hence (5.63) becomes $g=\lambda^{-2} \eta^{2}(x) \mathrm{d} x^{2}+\varphi^{-2} k^{2}(y) \mathrm{d} y^{2}$. So, if $u(x)$ and $v(y)$ are anti-derivatives of $\eta(x)$ and $k(y)$, respectively, then we obtain

$$
\begin{aligned}
& g=\lambda^{-2} \mathrm{~d} u^{2}+\varphi^{-2} \mathrm{~d} v^{2}, \\
& h\left(\frac{\partial}{\partial u}, \frac{\partial}{\partial u}\right)=J \frac{\partial}{\partial u}, \quad h\left(\frac{\partial}{\partial u}, \frac{\partial}{\partial v}\right)=0, \quad h\left(\frac{\partial}{\partial v}, \frac{\partial}{\partial v}\right)=J \frac{\partial}{\partial v} .
\end{aligned}
$$

From (5.66) we have

$$
\left.\begin{array}{l}
\nabla_{\partial / \partial u} \frac{\partial}{\partial u}=-\frac{\lambda_{u}}{\lambda} \frac{\partial}{\partial u}+\frac{\varphi^{2} \lambda_{v}}{\lambda^{3}} \frac{\partial}{\partial v}, \\
\nabla_{\partial / \partial u} \frac{\partial}{\partial v}=-\frac{\lambda_{v}}{\lambda} \frac{\partial}{\partial u}-\frac{\varphi_{u}}{\varphi} \frac{\partial}{\partial v}, \\
\nabla_{\partial / \partial v} \frac{\partial}{\partial v}=\frac{\lambda^{2} \varphi_{u}}{\varphi^{3}} \frac{\partial}{\partial u}-\frac{\varphi_{v}}{\varphi} \frac{\partial}{\partial v} .
\end{array}\right\}
$$


Also, from (5.65) and (5.67), we find

$$
\lambda \omega_{1}^{2}\left(e_{1}\right)=\varphi \lambda_{v}, \quad \varphi \omega_{2}^{1}\left(e_{2}\right)=\lambda \varphi_{u}, \quad \varphi^{3} \lambda_{v}=\lambda^{3} \varphi_{u} .
$$

Since $M$ is flat, (5.67) and (5.69) imply that

$$
\left(\varphi \lambda^{-2} \lambda_{v}\right)_{v}+\left(\lambda \varphi^{-2} \varphi_{u}\right)_{u}=0 .
$$

Thus, if we put $f=\varphi \lambda_{v} / \lambda^{2}=\lambda \varphi_{u} / \varphi^{2}$, we obtain

$$
\varphi \lambda^{-2} \lambda_{v}=\lambda \varphi^{-2} \varphi_{u}=f(u-v) .
$$

Also, from the last equation of (5.69), we have

$$
\left(\lambda^{-2}\right)_{v}=\left(\varphi^{-2}\right)_{u} .
$$

By applying (5.67)-(5.69) and Gauss's formula, we obtain

$$
\left.\begin{array}{rl}
L_{u u} & =\left(\mathrm{i}-(\ln \lambda)_{u}\right) L_{u}+(\ln \varphi)_{u} L_{v}, \\
L_{u v} & =-(\ln \lambda)_{v} L_{u}-(\ln \varphi)_{u} L_{v}, \\
L_{v v} & =(\ln \lambda)_{v} L_{u}+\left(i-(\ln \varphi)_{v}\right) L_{v} .
\end{array}\right\}
$$

Case B.2.i.a. $\mu=0$ and $\lambda=\varphi$. In this case, (5.72) reduces to $\lambda_{u}=\lambda_{v}$. Thus $\lambda=\varphi$ is a function of $w=u+v$. Hence, (5.70) yields $(\ln f)^{\prime \prime}(w)=0$, which implies that $\lambda=c \mathrm{e}^{a(u+v)}$ for some constants $a, c$. Therefore, system (5.73) reduces to

$$
\left.\begin{array}{rl}
L_{u u} & =(\mathrm{i}-a) L_{u}+a L_{v}, \\
L_{u v} & =-a L_{u}-a L_{v} \\
L_{v v} & =a L_{u}+(\mathrm{i}-a) L_{v} .
\end{array}\right\}
$$

From (5.74) we get $L_{u u}+L_{u v}=(\mathrm{i}-2 a) L_{u}, L_{v v}+L_{u v}=(\mathrm{i}-2 a) L_{v}$, which give

$$
\left.\begin{array}{l}
L_{u}+L_{v}=(\mathrm{i}-2 a) L+H(v), \\
L_{u}+L_{v}=(\mathrm{i}-2 a) L+F(u)
\end{array}\right\}
$$

for some functions $F(u), H(v)$. Comparing the two equations in (5.75) gives $H(v)=F(u)$. Thus $H=F$ is constant. By putting $H=(\mathrm{i}-2 a) c_{3}$ and substituting (5.75) into the first equation of (5.74), we get $L_{u u}=(\mathrm{i}-2 a)\left(L_{u}+a L+a c_{3}\right)$. Hence we obtain

$$
L=\exp \{(\mathrm{i}-2 a) u / 2\}\left(A(v) \exp \left\{\sqrt{1+4 a^{2}} u / 2\right\}+B(v) \exp \left\{-\sqrt{1+4 a^{2}} u / 2\right\}\right)-c_{3}
$$

for some vector functions $A, B$. We may choose $c_{3}=0$ by applying a suitable translation on $\mathbb{C}^{2}$. So, substituting (5.76) into the first equation of (5.74) gives

$$
\begin{aligned}
& 2 A^{\prime}(v)=\left(\mathrm{i}-2 a+\mathrm{i} \sqrt{1+4 a^{2}}\right) A(v), \\
& 2 B^{\prime}(v)=\left(\mathrm{i}-2 a-\mathrm{i} \sqrt{1+4 a^{2}}\right) B(v) .
\end{aligned}
$$


Solving these two equations yields

$$
L=\exp \{(\mathrm{i}-2 a)(u+v) / 2\}\left(c_{1} \cos \left(\frac{1}{2} \sqrt{1+4 a^{2}}(u-v)\right)+c_{2} \sin \left(\frac{1}{2} \sqrt{1+4 a^{2}}(u-v)\right)\right) .
$$

Hence, after choosing suitable initial conditions, we obtain

$$
L=\frac{\sqrt{2} \mathrm{e}^{(\mathrm{i}-2 a)(u+v) / 2}}{c \sqrt{1+a^{2}}}\left(\cos \left(\frac{1}{2} \sqrt{1+4 a^{2}}(u-v)\right), \sin \left(\frac{1}{2} \sqrt{1+4 a^{2}}(u-v)\right)\right),
$$

where $c \neq 0$. Thus we obtain (8) after putting $s=(u+v) / 2$ and $t=(u-v) / 2$.

Case B.2.i.b. $\mu=0$ and $\lambda \neq \varphi$. In this case (5.65) implies that $e_{2} \lambda, e_{1} \varphi, \omega_{1}^{2}\left(e_{2}\right)$ are non-zero. If we put $\rho=1 / \lambda$, then (5.71) gives

$$
\rho_{v} \varphi=-f(u-v) .
$$

Hence, (5.66) becomes

$$
g=\rho^{2} \mathrm{~d} u \otimes \mathrm{d} u+\frac{\rho_{v}^{2}}{f^{2}(u-v)} \mathrm{d} v \otimes \mathrm{d} v,
$$

which implies that

$$
\left.\begin{array}{l}
\nabla_{\partial / \partial u} \frac{\partial}{\partial u}=\frac{\rho_{u}}{\rho} \frac{\partial}{\partial u}-\frac{\rho f^{2}}{\rho_{v}} \frac{\partial}{\partial v}, \\
\nabla_{\partial / \partial u \frac{\partial}{\partial v}}=\frac{\rho_{v}}{\rho} \frac{\partial}{\partial u}+\left(\frac{\rho_{u v}}{\rho_{v}}-\frac{f^{\prime}}{f}\right) \frac{\partial}{\partial v}, \\
\nabla_{\partial / \partial v} \frac{\partial}{\partial v}=\left(\frac{\rho_{v}^{2} f^{\prime}}{\rho^{2} f^{3}}-\frac{\rho_{v} \rho_{u v}}{\rho^{2} f^{2}}\right) \frac{\partial}{\partial u}+\left(\frac{\rho_{v v}}{\rho_{v}}+\frac{f^{\prime}}{f}\right) \frac{\partial}{\partial v}, \quad f=f(u-v) .
\end{array}\right\}
$$

From (5.77) we obtain

$$
\varphi_{u}=\frac{\rho_{u v} f}{\rho_{v}^{2}}-\frac{f^{\prime}(u-v)}{\rho_{v}} .
$$

Substituting this into the last equation of (5.69) gives

$$
\left(\rho_{v}\right)^{-2} \rho_{u v} f(u-v)-\left(\rho_{v}\right)^{-1} f^{\prime}(u-v)=\varphi^{2} f(u-v) \rho .
$$

Combining this with (5.77) implies that $\rho$ satisfies the wave equation:

$$
f(u-v) \rho_{u v}-f^{\prime}(u-v) \rho_{v}-f^{3}(u-v) \rho=0 .
$$

From (5.67) and (5.78)-(5.80) we obtain

$$
\left.\begin{array}{l}
L_{u u}=\left(\mathrm{i}+\frac{\rho_{u}}{\rho}\right) L_{u}-\frac{\rho}{\rho_{v}} f^{2} L_{v}, \\
L_{u v}=\frac{\rho_{v}}{\rho} L_{u}+\frac{\rho}{\rho_{v}} f^{2} L_{v}, \\
L_{v v}=-\frac{\rho_{v}}{\rho} L_{u}+\left(\mathrm{i}+\frac{\rho_{v v}}{\rho_{v}}+\frac{f^{\prime}}{f}\right) L_{v}, \quad f=f(u-v) .
\end{array}\right\}
$$


Combining the first and the second equations in (5.81) gives

$$
L_{u u}+L_{u v}=\left(\mathrm{i}+\frac{\rho_{u}}{\rho}+\frac{\rho_{v}}{\rho}\right) L_{u}
$$

which gives $L_{u}=G(u-v) \rho \mathrm{e}^{\mathrm{i}(u+v) / 2}$. If we put $G(u-v)=z(u-v) \mathrm{e}^{\mathrm{i}(u-v) / 2}$, we find

$$
L_{u}=z(u-v) \rho \mathrm{e}^{\mathrm{i} u}
$$

On the other hand, by (5.80) and the last two equations of (5.81), we find

$$
L_{u v}+L_{v v}=\left(\mathrm{i}+\frac{\rho_{u v}}{\rho_{v}}+\frac{\rho_{v v}}{\rho_{v}}\right) L_{v}
$$

Solving this differential equation gives $L_{v}=H(u-v) \rho_{v} \mathrm{e}^{\mathrm{i}(u+v) / 2}$, where $H$ is a positive function. So, if we put $H(u-v)=F(u-v) \mathrm{e}^{\mathrm{i}(u-v) / 2}$, we obtain

$$
L_{v}=F(u-v) \rho_{v} \mathrm{e}^{\mathrm{i} u} .
$$

By taking the derivative of (5.83) with respect to $v$ and comparing it with (5.85) and the second equation of (5.81), we find $z^{\prime}=-f^{2} F$. Therefore, (5.85) becomes

$$
L_{v}=-\frac{\rho_{v} z^{\prime}}{f^{2}} \mathrm{e}^{\mathrm{i} u}
$$

Also, after taking the derivative of this equation with respect to $u$ and comparing it with the second equation of (5.81), we obtain

$$
z^{\prime \prime}+\left(\mathrm{i}-\frac{f^{\prime}}{f}\right) z^{\prime}+f^{2} z=0
$$

by virtue of (5.79), (5.83) and (5.86). Integrating (5.83) with respect to $u$ gives

$$
L(u, v)=\int_{0}^{u} z(u-v) \rho \mathrm{e}^{\mathrm{i} u} \mathrm{~d} u+H(v) .
$$

Differentiating this with respect to $v$ and comparing it with (5.86) yield

$$
H^{\prime}(v)=-\frac{\rho_{v} z^{\prime}}{f^{2}} \mathrm{e}^{\mathrm{i} u}-\int_{0}^{u} \mathrm{e}^{\mathrm{i} u}(z(u-v) \rho)_{v} \mathrm{~d} u .
$$

Thus $L$ is congruent to

$$
L(u, v)=\int_{0}^{u} \rho z(u-v) \mathrm{e}^{\mathrm{i} u} \mathrm{~d} u-\int_{0}^{v}\left\{\frac{\rho_{v} z^{\prime}(u-v)}{f^{2}} \mathrm{e}^{\mathrm{i} u}+\int_{0}^{u} \mathrm{e}^{\mathrm{i} u}(\rho z(u-v))_{v} \mathrm{~d} u\right\} \mathrm{d} v .
$$

Using (5.78), (5.83) and (5.86), we find $|z|^{2}=1,\left|z^{\prime}\right|^{2}=f^{2}$. Thus $z$ is a curve in $S^{3}(1) \subset \mathbb{C}^{2}$ with $f$ as its speed. Also, it follows from (5.87) and Lemma 3.1 that $z$ is a Legendre curve with curvature function $-f^{-1}$. Therefore, we obtain (9). 
Case B.2.ii. $\mu \neq 0$. We divide this case into two cases: $\lambda=\mu$ and $\lambda \neq \mu$.

Case B.2.ii.a. $\mu \neq 0$ and $\lambda=\mu$. If $\theta$ is a solution of $\lambda\left(1-2 \cos ^{2} \theta\right)=\varphi \sin \theta \cos \theta$ and $\hat{e}_{1}=\cos \theta e_{1}+\sin \theta e_{2}, \hat{e}_{2}=-\sin \theta e_{1}+\cos \theta e_{2}$, then (5.1) yields

$$
h\left(\hat{e}_{1}, \hat{e}_{1}\right)=\hat{\lambda} J \hat{e}_{1}, \quad h\left(\hat{e}_{1}, \hat{e}_{1}\right)=0, \quad h\left(\hat{e}_{2}, \hat{e}_{2}\right)=\hat{\varphi} J \hat{e}_{2},
$$

where

$$
\hat{\lambda}=\sin ^{2} \theta(2 \lambda \cos \theta+\varphi \sin \theta)+\lambda \cos \theta, \quad \hat{\varphi}=\cos ^{2} \theta(\varphi \cos \theta-2 \lambda \sin \theta)-\lambda \sin \theta .
$$

So, this case reduces to Case B.1.ii.a or B.2.i according to $\nabla_{e_{1}} e_{1}=0$ or $\nabla_{e_{1}} e_{1} \neq 0$.

Case B.2.ii.b. $\mu \neq 0$ and $\lambda \neq \mu$. From the assumption $\nabla_{e_{1}} e_{1} \neq 0$ for Case B and the second equation in (5.2), we get $e_{2} \lambda \neq 0$. Since $K=\lambda \mu-\mu^{2} \neq 0$, we have

$$
\mu e_{j} \lambda=(2 \mu-\lambda) e_{j} \mu, \quad j=1,2,
$$

which implies $e_{2} \mu \neq 0$ by $\lambda \neq 2 \mu$. Combining (5.2) with (5.91) gives

$$
\left.\begin{array}{rl}
e_{1} \mu & =\varphi \omega_{1}^{2}\left(e_{1}\right)+(\lambda-2 \mu) \omega_{1}^{2}\left(e_{2}\right), \\
e_{1} \varphi & =4 \mu \omega_{2}^{1}\left(e_{1}\right)+\varphi \omega_{2}^{1}\left(e_{2}\right), \\
(\ln \mu) & =\omega_{2}^{1}\left(e_{1}\right) .
\end{array}\right\}
$$

Since $K=\lambda \mu-\mu^{2}$, the first two equations in (5.92) imply that

$$
4 \mu e_{1} \mu+\varphi e_{1} \varphi=\left(4 \lambda \mu-8 \mu^{2}-\varphi^{2}\right) \omega_{1}^{2}\left(e_{2}\right)=\left(4 K-4 \mu^{2}-\varphi^{2}\right) \omega_{1}^{2}\left(e_{2}\right) .
$$

Case B.2.ii.b. $\alpha .4 K=4 \mu^{2}+\varphi^{2}$. We have $0 \neq \varphi^{2}=4\left(K-\mu^{2}\right)$, which gives $K>\mu^{2}$. So, we may put $\varphi=2 \sqrt{K-\mu^{2}}$. Thus we obtain from $K=\lambda \mu-\mu^{2}$ and (5.92) that

$$
e_{1} \mu=\frac{K-\mu^{2}}{\mu} \omega_{1}^{2}\left(e_{2}\right)-2 \sqrt{K-\mu^{2}} e_{2}(\ln \mu) .
$$

The last equation of (5.92) and the structure equation yield $\mathrm{d}\left(\mu^{-1} \omega^{1}\right)=0$. Thus there exists a function $u$ with $\mathrm{d} u=\mu^{-1} \omega^{1}, \partial / \partial u=\mu e_{1}$. Let $\Phi$ be a function satisfying

$$
e_{2} \mu^{2}=\sqrt{K-\mu^{2}}(\ln \Phi)_{u} .
$$

By applying $\partial / \partial u=\mu e_{1},(5.94),(5.95)$ and the last equation in (5.92), it is easy to verify that

$$
\left[\frac{\partial}{\partial u}, \frac{\Phi e_{2}}{\sqrt{K-\mu^{2}}}\right]=0
$$

Therefore, there exists a function $v$ such that $\partial / \partial v=\Phi e_{2} / \sqrt{K-\mu^{2}}$. With respect to such a coordinate system $\{u, v\}$, we have

$$
g=\mu^{2} \mathrm{~d} u^{2}+\frac{\Phi^{2}}{K-\mu^{2}} \mathrm{~d} v^{2}, \quad \frac{\partial \Phi}{\partial u}=\frac{\partial \mu^{2}}{\partial v},
$$


which implies that

$$
\left.\begin{array}{l}
\nabla_{\partial / \partial u} \frac{\partial}{\partial u}=(\ln \mu)_{u} \frac{\partial}{\partial u}-\frac{\left(K-\mu^{2}\right) \mu \mu_{v}}{\Phi^{2}} \frac{\partial}{\partial v}, \\
\nabla_{\partial / \partial u \frac{\partial}{\partial v}}=(\ln \mu)_{v} \frac{\partial}{\partial u}+\left(\frac{\mu \mu_{u}}{K-\mu^{2}}+(\ln \Phi)_{u}\right) \frac{\partial}{\partial v}, \\
\nabla_{\partial / \partial v \frac{\partial}{\partial v}}=-\frac{\Phi^{2} \mu \mu_{u}+\left(K-\mu^{2}\right) \Phi \Phi_{u}}{\mu^{2}\left(K-\mu^{2}\right)^{2}} \frac{\partial}{\partial u}+\left(\frac{\mu \mu_{v}}{K-\mu^{2}}+(\ln \Phi)_{v}\right) \frac{\partial}{\partial v} .
\end{array}\right\}
$$

Using (5.96) we know that the Gauss curvature $K$ and $\mu$ are related by

$$
K=-\frac{\sqrt{K-\mu^{2}}}{\mu \Phi}\left\{\left(\frac{\mu_{v} \sqrt{K-\mu^{2}}}{\Phi}\right)_{v}+\left(\frac{2\left(K-\mu^{2}\right) \mu_{v}+\Phi \mu_{u}}{\left(K-\mu^{2}\right)^{3 / 2}}\right)_{u}\right\} .
$$

Thus, by (5.1), (5.96), (5.98), $K=\lambda \mu-\mu^{2}$ and Gauss's formula, we obtain

$$
\begin{aligned}
L_{u u} & =\left\{\mathrm{i}\left(K+\mu^{2}\right)+(\ln \mu)_{u}\right\} L_{u}-\frac{\left(K-\mu^{2}\right) \mu \mu_{v}}{\Phi^{2}} L_{v}, \\
L_{u v} & =(\ln \mu)_{v} L_{u}+\left\{\mathrm{i} \mu^{2}+\frac{\mu \mu_{u}}{K-\mu^{2}}+\frac{2 \mu \mu_{v}}{\Phi}\right\} L_{v}, \\
L_{v v} & =\left\{\frac{\mathrm{i} \Phi}{K-\mu^{2}}-\frac{\Phi \mu_{u}+2\left(K-\mu^{2}\right) \mu_{v}}{\mu\left(K-\mu^{2}\right)^{2}}\right\} \Phi L_{u}+\left\{2 \mathrm{i} \Phi+\frac{\mu \mu_{v}}{K-\mu^{2}}+\frac{\Phi}{\Phi}\right\} L_{v} .
\end{aligned}
$$

A direct computation shows that the compatibility conditions: $L_{u u v}=L_{u v u}$ and $L_{u v v}=L_{v v u}$ hold if and only if (5.96) and (5.98) hold true. Thus we obtain (17).

Case B.2.ii.b. $\boldsymbol{\beta} .4 K \neq 4 \mu^{2}+\varphi^{2}$. From (5.91) and (5.93) we obtain $\omega_{1}^{2}\left(e_{2}\right)=e_{1}(\ln G)$ and $G=1 / \sqrt{\left|4 K-4 \mu^{2}-\varphi^{2}\right|}$. So, we have $\left[\mu e_{1}, G e_{2}\right]=0$. Thus, there exist local coordinates $\{u, v\}$ with $\partial / \partial u=\mu e_{1}, \partial / \partial v=G e_{2}$ such that the metric is given by

$$
g=\mu^{2} \mathrm{~d} u^{2}+\frac{\mathrm{d} v^{2}}{\left|4 K-4 \mu^{2}-\varphi^{2}\right|} .
$$

If $4 K>4 \mu^{2}+\varphi^{2}$, then (5.100) implies that

$$
\left.\begin{array}{l}
\nabla_{\partial / \partial u} \frac{\partial}{\partial u}=(\ln \mu)_{u} \frac{\partial}{\partial u}-\left(4 K-4 \mu^{2}-\varphi^{2}\right) \mu \mu_{v} \frac{\partial}{\partial v}, \\
\nabla_{\partial / \partial u} \frac{\partial}{\partial v}=(\ln \mu)_{v} \frac{\partial}{\partial u}+\frac{4 \mu \mu_{u}+\varphi \varphi_{u}}{4 K-4 \mu^{2}-\varphi^{2}} \frac{\partial}{\partial v}, \\
\nabla_{\partial / \partial v} \frac{\partial}{\partial v}=-\frac{4 \mu \mu_{u}+\varphi \varphi_{u}}{\mu^{2}\left(4 K-4 \mu^{2}-\varphi^{2}\right)^{2}} \frac{\partial}{\partial u}+\frac{4 \mu \mu_{v}+\varphi \varphi_{v}}{4 K-4 \mu^{2}-\varphi^{2}} \frac{\partial}{\partial v} .
\end{array}\right\}
$$


From (5.1), (5.3), (5.101) and Gauss's formula, we have

$$
\left.\begin{array}{rl}
L_{u u}= & \left\{\mathrm{i} K+\mathrm{i} \mu^{2}+(\ln \mu)_{u}\right\} L_{u}-\left(4 K-4 \mu^{2}-\varphi^{2}\right) \mu \mu_{v} L_{v}, \\
L_{u v}= & (\ln \mu)_{v} L_{u}+\left\{\mathrm{i} \mu^{2}+\frac{4 \mu \mu_{u}+\varphi \varphi_{u}}{4 K-4 \mu^{2}-\varphi^{2}}\right\} L_{v}, \\
L_{v v}= & \left\{\frac{\mathrm{i}}{4 K-4 \mu^{2}-\varphi^{2}}-\frac{4 \mu \mu_{u}+\varphi \varphi_{u}}{\mu^{2}\left(4 K-4 \mu^{2}-\varphi^{2}\right)^{2}}\right\} L_{u} \\
& +\left\{\frac{\mathrm{i} \varphi}{\sqrt{4 K-4 \mu^{2}-\varphi^{2}}}+\frac{4 \mu \mu_{v}+\varphi \varphi_{v}}{4 K-4 \mu^{2}-\varphi^{2}}\right\} L_{v} .
\end{array}\right\}
$$

By a straightforward long computation we know that the compatibility conditions $\left(L_{u u}\right)_{v}=\left(L_{u y}\right)_{u}$ and $\left(L_{u y}\right)_{v}=\left(L_{v v}\right)_{u}$ hold if and only if $\mu$ and $\varphi$ satisfy

$$
\mu_{v}=\frac{K \varphi_{u}+\varphi \mu \mu_{u}-\mu^{2} \varphi_{u}}{\mu\left(4 K-4 \mu^{2}-\varphi^{2}\right)^{3 / 2}}, \quad\left(\frac{G_{u}}{\mu}\right)_{u}+\left(\frac{\mu_{v}}{G}\right)_{v}=-K \mu G,
$$

where $G=1 / \sqrt{4 K-4 \mu^{2}-\varphi^{2}}$. From these we obtain (18).

If $4 K<4 \mu^{2}+\varphi^{2},(5.100)$ gives

$$
\left.\begin{array}{l}
\nabla_{\partial / \partial u} \frac{\partial}{\partial u}=(\ln \mu)_{u} \frac{\partial}{\partial u}+\left(4 K-4 \mu^{2}-\varphi^{2}\right) \mu \mu_{v} \frac{\partial}{\partial v}, \\
\nabla_{\partial / \partial u} \frac{\partial}{\partial v}=(\ln \mu)_{v} \frac{\partial}{\partial u}+\frac{4 \mu \mu_{u}+\varphi \varphi_{u}}{4 K-4 \mu^{2}-\varphi^{2}} \frac{\partial}{\partial v}, \\
\nabla_{\partial / \partial v} \frac{\partial}{\partial v}=\frac{4 \mu \mu_{u}+\varphi \varphi_{u}}{\mu^{2}\left(4 K-4 \mu^{2}-\varphi^{2}\right)^{2}} \frac{\partial}{\partial u}+\frac{4 \mu \mu_{v}+\varphi \varphi_{v}}{4 K-4 \mu^{2}-\varphi^{2}} \frac{\partial}{\partial v} .
\end{array}\right\}
$$

From (5.1), (5.3), and (5.104) we have

$$
\left.\begin{array}{rl}
L_{u u}= & \left\{\mathrm{i} K+\mathrm{i} \mu^{2}+(\ln \mu)_{u}\right\} L_{u}+\left(4 K-4 \mu^{2}-\varphi^{2}\right) \mu \mu_{v} L_{v}, \\
L_{u v}= & (\ln \mu)_{v} L_{u}+\left\{\mathrm{i} \mu^{2}+\frac{4 \mu \mu_{u}+\varphi \varphi_{u}}{4 K-4 \mu^{2}-\varphi^{2}}\right\} L_{v}, \\
L_{v v}= & \left\{\frac{\mathrm{i}}{4 K-4 \mu^{2}-\varphi^{2}}+\frac{4 \mu \mu_{u}+\varphi \varphi_{u}}{\mu^{2}\left(4 K-4 \mu^{2}-\varphi^{2}\right)^{2}}\right\} L_{u} \\
& +\left\{\frac{\mathrm{i} \varphi}{\sqrt{\varphi^{2}-4 K+4 \mu^{2}}}+\frac{4 \mu \mu_{v}+\varphi \varphi_{v}}{4 K-4 \mu^{2}-\varphi^{2}}\right\} L_{v} .
\end{array}\right\}
$$

A straightforward computation implies that the compatibility conditions: $\left(L_{u u}\right)_{v}=$ $\left(L_{u y}\right)_{u}$ and $\left(L_{u y}\right)_{v}=\left(L_{v v}\right)_{u}$ hold if and only if $\mu$ and $\varphi$ satisfy

$$
\mu_{v}=\frac{\mu^{2} \varphi_{u}-K \varphi_{u}-\varphi \mu \mu_{u}}{\mu\left(4 \mu^{2}+\varphi^{2}-4 K\right)^{3 / 2}}, \quad\left(\frac{G_{u}}{\mu}\right)_{u}+\left(\frac{\mu_{v}}{G}\right)_{v}=-K \mu G
$$

where $G=1 / \sqrt{4 \mu^{2}+\varphi^{2}-4 K}$. From these we obtain (19). 


\section{Remarks}

Remark 6.1. By a very long straightforward computation, we know that the surfaces given in Theorem 5.1 are Lagrangian surfaces of constant curvature.

Remark 6.2. There exist Lagrangian surfaces in $\mathbb{C}^{2}$ which do not contain any Legendre curve in any hypersphere of $\mathbb{C}^{2}$. The simplest example is the Lagrangian circular cylinder: $L(s, t)=\left(\mathrm{e}^{\mathrm{i} s}, t\right), s, t \in \mathbb{R}$. On the other hand, we see from Theorem 5.1 that 12 of the 19 families of Lagrangian surfaces of constant curvature in $\mathbb{C}^{2}$ given in Theorem 5.1 can be constructed via Legendre curves.

Remark 6.3. For a non-zero real-valued function $f$ of one variable, the wave equation

$$
f(u-v) \rho_{u v}-f^{\prime}(u-v) \rho_{v}-f^{3}(u-v) \rho=0
$$

admits infinitely many solutions. For example, every linear combination of

$$
\rho_{1}=\sin \left(\int_{0}^{u-v} f(t) \mathrm{d} t\right), \quad \rho_{2}=\cos \left(\int_{0}^{u-v} f(t) \mathrm{d} t\right)
$$

is a solution of $(6.1)$ (see $[\mathbf{1 1}$, p. 738]). Consequently, the family of Lagrangian surfaces given in (9) of Theorem 5.1 is very large.

Remark 6.4. Locally, there exist many solutions of system (4.1) (respectively, (4.5) or (4.9)) according to the Cauchy-Kowaleski theorem (see [20]). Hence there exist many Lagrangian surfaces of constant curvature $K$ whose second fundamental forms are given by (4.2) (respectively, given by (4.6) or (4.10)). Hence the families of Lagrangian surfaces of constant curvature given in (17)-(19) of Theorem 5.1 are large.

Remark 6.5. The complete classifications of Lagrangian surfaces of constant curvature in complex projective plane and in complex hyperbolic plane are obtained in $[\mathbf{1 3}]$ and $[\mathbf{1 4}]$, respectively.

\section{References}

1. R. Aiyama, Lagrangian surfaces in the complex 2-space, in Proc. 5th Int. Workshop Differential Geometry, Taegu, 2000, pp. 25-29 (Kyungpook National University, Taegu, 2001).

2. D. E. BlaIR, Lagrangian helicoids, Michigan Math. J. 50 (2002), 187-200.

3. I. Castro and C. R. Montealegre, A family of surfaces with constant curvature in Euclidean four-space, Soochow J. Math. 30 (2004), 293-301.

4. I. Castro and F. URbano, Lagrangian surfaces in the complex Euclidean plane with conformal Maslov form, Tôhoku Math. J. 45 (1993), 656-582

5. I. Castro and F. Urbano, On a minimal Lagrangian submanifold of $\mathbb{C}^{n}$ foliated by spheres, Michigan Math. J. 46 (1998), 71-82.

6. I. CASTRo and F. URBAno, Examples of unstable Hamiltonian-minimal Lagrangian tori in $\mathbb{C}^{2}$, Compositio Math. 111 (1998), 1-14.

7. B.-Y. Chen, Geometry of submanifolds (Dekker, New York, 1973).

8. B.-Y. CHEN, Interaction of Legendre curves and Lagrangian submanifolds, Israel J. Math. 99 (1997), 69-108. 
9. B.-Y. CHEN, Representation of flat Lagrangian $H$-umbilical submanifolds in complex Euclidean spaces, Tôhoku Math. J. 51 (1999), 13-20.

10. B.-Y. Chen, Riemannian geometry of Lagrangian submanifolds, Taiwan. J. Math. 5 (2001), 681-723.

11. B.-Y. CHEN, Classification of flat slant surfaces in complex Euclidean plane, J. Math. Soc. Jpn 54 (2002), 719-746.

12. B.-Y. Chen, Lagrangian surfaces of constant curvature in complex Euclidean plane, Tôhoku Math. J. 56 (2004), 289-298.

13. B.-Y. CHEN, Classification of Lagrangian surfaces of constant curvature in complex projective planes, J. Geom. Phys. 53 (2005), 428-460.

14. B.-Y. CHEN, Classification of Lagrangian surfaces of constant curvature in complex hyperbolic planes, in the press. J. Geom. Phys. (2005), in press.

15. B.-Y. CHEN, Construction of Lagrangian surfaces in complex Euclidean plane with Legendre curves, Preprint.

16. B.-Y. Chen And O. J. GARAY, Maslovian Lagrangian isometric immersions of real space forms into complex space forms, Jpn. J. Math. 30 (2004), 227-281.

17. B.-Y. Chen And K. Ogiue, On totally real submanifolds, Trans. Am. Math. Soc. 193 (1974), 257-266.

18. B.-Y. Chen, F. Dillen, L. Verstraelen and L. Vrancken, An exotic totally real minimal immersion of $S^{3}$ in $C P^{3}$ and its characterization, Proc. R. Soc. Edinb. A 126 (1996), 153-165.

19. B.-Y. Chen, F. Dillen, L. Verstraelen and L. Vrancken, Lagrangian isometric immersions of a real-space-form $M^{n}(c)$ into a complex-space-form $\tilde{M}^{n}(4 c)$, Math. Proc. Camb. Phil. Soc. 124 (1998), 107-125.

20. R. Courant And D. Hilbert, Methods of mathematical physics (Wiley-Interscience, 1962).

21. N. EJIRI, Totally real minimal immersions of $n$-dimensional totally real space forms into n-dimensional complex space forms, Proc. Am. Math. Soc. 84 (1982), 243-246.

22. F. Helein AND P. Romon, Weierstrass representation of Lagrangian surfaces in fourdimensional space using spinors and quaternions, Comment. Math. Helv. 75 (2000), 668680 .

23. J. D. Moore And J.-M. Morvan, On isometric Lagrangian immersions, Illinois J. Math. 45 (2001), 833-849. 